\title{
ATGL deficiency aggravates pressure overload-triggered myocardial hypertrophic remodeling associated with the proteasome-PTEN-mTOR-autophagy pathway
}

\author{
Xiao Han · Yun-Long Zhang • Qiu-Yue Lin • \\ Hui-Hua Li (D) Shu-Bin Guo
}

Received: 24 June 2021 / Accepted: 26 January 2022

(C) The Author(s) 2022

\begin{abstract}
Persistent myocardial hypertrophy frequently leads to heart failure (HF). Intramyocardial triacylglycerol (TAG) accumulation is closely related with cardiac remodeling and abnormal contractile function. Adipose triglyceride lipase (ATGL), a key enzyme in TAG metabolism, regulates cardiac function. However, its associated molecular pathways have not been fully defined. Here, cardiac hypertrophy and HF were induced in wild-type (WT) or ATGL knockout (KO) mice through transverse aortic constriction (TAC) for up to 4 weeks. TAC in WT mice significantly reduced cardiac function and
\end{abstract}

Headlights 1. Pressure overload reduces ATGL expression in the heart.

2. Deficiency of ATGL accelerates pressure overloadinduced cardiac hypertrophy and dysfunction.

3. Knockout of ATGL upregulates expression and activity of the proteasome catalytic subunits, which promotes PTEN degradation leading to activation of mTOR signaling and inhibition of autophagy.

4. Blocking proteasome activity or activating autophagy ameliorates cardiac hypertrophy and dysfunction in ATGL knockout mice.

Supplementary Information The online version contains supplementary material available at https://doi. org/10.1007/s 10565-022-09699-0.

X. Han · Y.-L. Zhang · H.-H. Li $(\bowtie) \cdot$ S.-B. Guo $(\bowtie)$ Department of Emergency Medicine, Beijing Key Laboratory of Cardiopulmonary Cerebral Resuscitation, Beijing Chaoyang Hospital, Capital Medical University, Beijing 100020, China

e-mail: hhli1995@yahoo.com autophagy while enhancing left ventricular hypertrophy, interstitial fibrosis, inflammatory response, superoxide generation, and cardiomyocyte apoptosis, accompanied with upregulation of the proteasome activity, reduction of PTEN level and activation of AKT-mTOR signaling, and these effects were further aggravated in ATGL KO mice. Interestingly, ATGL KO-mediated cardiac dysfunction and remodeling were markedly reversed by proteasome inhibitor (epoxomicin) or autophagic activator (rapamycin), but accelerated by PTEN inhibitor (VO-OHpic) or autophagy inhibitor 3-MA. Mechanistically, ATGL KO upregulated proteasome expression and activity, which in turn mediates PTEN degradation leading to activation of AKT-mTOR signaling and inhibition of autophagy, thereby enhancing hypertrophic remodeling and HF. In conclusion, ATGL KO contributes to TAC-induced cardiac dysfunction and adverse remodeling probably associated with the proteasomePTEN-mTOR-autophagy pathway. Therefore, modulation of this pathway may have a therapeutic effect potential for hypertrophic heart disease.

S.-B. Guo

e-mail: shubinguo@126.com

Q.-Y. Lin

Department of Cardiology, Institute of Cardiovascular Diseases, First Affiliated Hospital of Dalian Medical University, Dalian 116011, China 
Keywords ATGL · Cardiac remodeling ·

Proteasome $\cdot$ PTEN $\cdot$ mTOR $\cdot$ Autophagy

\section{Introduction}

Sustained pressure overload frequently triggers myocardial hypertrophy and eventually resulting in heart failure (HF). Cardiac hypertrophy presents with activation of fetal gene expression, protein synthesis, increased cell size, and actin reorganization (Heineke and Molkentin 2006). Several signaling pathways, including PTEN/AKT/mTOR, MAPK, and calcineurin/NFAT signaling pathways regulate cardiac hypertrophy (Heineke and Molkentin 2006). PTEN, a negative regulator of the AKT-mTOR signaling cascade, is essential in maintaining cardiac homeostasis, and its loss promotes pressure overload-induced cardiac pathological remodeling and contractile defects (Roe et al. 2015; Shi et al. 2020; Xu et al. 2014). Moreover, mTOR is a critical regulator of autophagy and cardiac hypertrophic signaling (Kim et al. 2018; Li et al. 2016; Shi et al. 2020; Xu et al. 2014). Therefore, modulation of PTEN/mTOR signaling may have therapeutic effects against cardiac hypertrophy and dysfunction.

Myocardial triacylglycerol (TAG), which is stored in cytosolic lipid droplets, is a highly dynamic fatty acid storage pool and energy source of the heart. In patients with hypertrophic heart disease, myocardial TG level is negatively associated with cardiac left ventricle (LV) mass and stroke volume in patients with cardiac hypertrophy (Sai et al. 2017). In multiple tissues, including the heart, ATGL (adipose triglyceride lipase or PNPLA2) regulates TAG lipolysis by hydrolyzing fatty acids from TAG stores (Kintscher et al. 2020). ATGL is highly expressed in various tissues, including brown and white adipose tissues, muscles, and heart tissues (Kintscher et al. 2020). Global knockout of the ATGL gene induces severe lipotrophic cardiomyopathy, leading to premature mortality (Haemmerle et al. 2006). Human ATGL mutations cause myocardial steatosis and dysfunction (Hirano et al. 2008). In mice models of cardiac hypertrophy and in in vitro phenylephrine-treated cardiomyocytes, ATGL levels were found to be suppressed (Gao et al. 2015), which regulated cardiac dysfunction and remodeling by affecting lipid and energy metabolism. ATGL knockout (KO) enhanced ceramide and intracellular free fatty acid accumulation, inducing by pressure overload stress (Kienesberger et al. 2012). Moreover, ATGL deficiency in mice suppressed PPAR- $\alpha$ and PGC-1 levels and severely impaired mitochondrial substrate oxidation, resulting in lipid deposition and cardiac dysfunction (Haemmerle et al. 2011). Myocardial ATGL KO alters TAG and energy metabolism, causing cardiac dysfunction. In contrast, overexpression of cardiacspecific ATGL markedly reduced myocardial TAG levels and the rate of fatty acid oxidation, preventing the occurrence of pressure overload-induced heart remodeling and systolic dysfunction (Kienesberger et al. 2012). However, it has not been established whether other molecular mechanisms regulate the effects of ATGL on pressure overloadinduced HF.

In this study, we showed that ATGL KO significantly enhances TAC-induced cardiac remodeling and $\mathrm{HF}$ associated with suppression of autophagy via the proteasome-PTEN-mTOR signaling. These findings uncover a new mechanism through which ATGL regulates cardiac function, implying that ATGL may represent a novel target for the treatment of hypertrophic disease.

\section{Material and methods}

Animals and treatment

C57BL/6 wild-type (WT) and global ATGL knockout (KO) mice (019,003-B6;129P2Pnpla2tm1Rze/J) were obtained from Jackson laboratory (Bar Harbor, ME, USA) and SPF Biotechnology Co., Ltd. (Beijing, China). In the ATGL KO strain, exon 1 of the Pnpla2 gene was replaced with a NEO cassette. The heterozygotes were viable and fertile, while the homozygotes exhibited a shortened lifespan (14-16 weeks) and died from severe cardiac steatosis and lethal myopathy (Haemmerle et al. 2006). Moreover, homozygotes exhibit impaired lipid homeostasis, glucose tolerance, and energy homeostasis (Trites and Clugston 2019). All animals were housed in a conditioned room with free access to receive sterilized standard mouse diet and drank water freely. 
Establishment of cardiac remodeling mice models and inhibitor administration

Cardiac hypertrophic mice models were established via transverse aortic constriction (TAC), as previously reported (Chen et al. 2019; Lin et al. 2021; Xie et al. 2019). Briefly, ATGL KO and WT mice (male, 8 weeks old, $n=8$ per group) were anesthetized by $2.0 \%$ isoflurane injection. Then, the left chest wall of the mice was opened the transverse aorta ligated (7.0 nylon suture), resulting in 65-70\% constriction after needle removal. Mice in the sham control group were received with the same procedures, but without ligation.

To activate or inhibit autophagy, animals $(n=8$ per group) were intraperitoneally injected with the mTOR inhibitor, rapamycin at $4 \mathrm{mg} / \mathrm{kg}$, or with 3-MA at $10 \mathrm{mg} / \mathrm{kg}$ daily for 4 weeks (Yang et al. 2020, 2021). For proteasome or PTEN inhibition, mice ( $n=6$ per group) were intraperitoneally injected with epoxomicin at $2.9 \mathrm{mg} / \mathrm{kg}$ or VO-OHpic at $10 \mathrm{mg} / \mathrm{kg}$ daily for 4 weeks (Han et al. 2020; Meng et al. 1999). These agents had been obtained from Selleck (Houston, TX, USA) and dissolved in $0.9 \%$ saline. Control mice were administered with similar volumes of saline. After treatment for 4 weeks, animals were anesthetized with $100 \mathrm{mg} / \mathrm{kg}$ sodium pentobarbital (SigmaAldrich, Dorset, UK), after which they were sacrificed, and their hearts obtained collected for further analyses.

\section{Echocardiographic assessment}

Four weeks after TAC, transthoracic echocardiographic measurements of mice were performed using a 30-MHz ultrasound system (Vevo 1100, VisualSonics, Toronto, ON, Canada). Several cardiac parameters were recorded as previously reported (Xie et al. 2019). The pulsed-wave Doppler was used for simultaneous recording of aortic arch blood velocities (Deng et al. 2021). Transaortic pressure gradients were analyzed by pulsed-wave doppler examination using the equation: Pressure gradient is equal to $4 \times$ velocity 2 . LV hemodynamics were determined using 1.4-F Millar pressure-volume catheters (SPR839) (Chen et al. 2019).
Histopathological examination

Mice were anesthetized using $100 \mathrm{mg} / \mathrm{kg}$ sodium pentobarbital (Sigma-Aldrich, Dorset, UK), heart tissues resected, fixed in $4 \%$ paraformaldehyde (PFA) for $24 \mathrm{~h}$, paraffin-embedded and then sliced to $5 \mu$ m-thick secrions. Staining of H\&E (haematoxylin-eosin) or Masson trichrome was performed on tissue slices (Chen et al. 2019; Xie et al. 2019; Zou et al. 2019). Cross-sectional areas of LV myocytes were evaluated by staining with rhodamine-labeled WGA (wheat germ agglutinin, $50 \mu \mathrm{g} / \mathrm{mL}$ ) for $60 \mathrm{~min}$ (Chen et al. 2019; Xie et al. 2019; Zou et al. 2019). Images were obtained from cardiac samples (>20 random fields per sample) at $\times 100$ magnification. Cardiac myocyte areas were determined by analysis of 200 cells per sample. Immunohistochemistry (IHC) was performed using antibodies against $\alpha$-smooth muscle actin ( $\alpha$-SMA) (Abcam, Cambridge, MA, USA) or Mac-2 (Santa Cruz, Dallas, Texas, USA). The oil red O staining assay was performed to assess lipid deposition on $10 \mu \mathrm{m}$-thick heart sections(Liao et al. 2020). Image Pro Plus 3.0 software was used for imaging analysis.

TUNEL staining for cardiomyocyte apoptosis

Cell apoptosis was detected by using the in situ cell death detection assay (Roche, Basel, Switzerland) according to protocols as described (Xie et al. 2019). The TUNEL kit was used to stain heart sections. Cardiomyocytes and nuclei were counterstained with antibodies against $\alpha$-actinin and DAPI (SigmaAldrich, Dorset, UK), respectively. Then, heart sections were imaged in 6-8 random fields of view with proportions of TUNEL-positive nuclei calculated as previously described (Xie et al. 2019).

Tissue and serum lipid analysis

Myocardial tissues were homogenized in a cold lysis buffer containing a protease inhibitors. Homogenates were cleared with centrifugation $(1,200 \times g)$ for $20 \mathrm{~min}$ at $4{ }^{\circ} \mathrm{C}$. Protein levels were measured with the BCA assay kit. Heart TG levels were evaluated using the TG assay kit (Solarbio, Beijing, China) while common serum lipoproteins were measured using the resuspended lipid extracts in ice-cold $1 \%$ Triton 
$\mathrm{X}-100$ via the colorimetric assay kit (Elabscience, Wuhan, China).

Quantitative real-time PCR analysis

Tissue RNAs were purified from cardiac left ventricles using the TRIzol buffer (Invitrogen, Carlsbad, CA, USA). Briefly, 1-2 $\mu \mathrm{g}$ cDNA was used for firststrand cDNA synthesis. qPCR was performed on a Bio-Rad iCycler IQ system (Chen et al. 2019; Xie et al. 2019; Zou et al. 2019) using gene-specific primers for interleukin (IL)-6, IL-1 $\beta$, BNP, ANF, Collagen I, and III. GAPDH was the reference gene. The primers used for qPCR analysis are illustrated in Supplemental Table 1.

\section{Immunoblotting analysis}

Total protein extracts were obtained from frozen heart tissues using the RIPA lysis buffer and quantified with the BCA assay method (Thermo Fisher, Carlsbad, CA, USA). Approximately 40-60 $\mu \mathrm{g}$ proteins were resolved on 8-10\% SDS-PAGE and transferred onto polyvinylidene fluoride membranes that were then blotted overnight with appropriate primary antibodies at $4{ }^{\circ} \mathrm{C}$ for $12 \mathrm{~h}$ (Chen et al. 2019; Xie et al. 2019; Zou et al. 2019). Then, membranes were developed on a Gel-pro Analyzer 4.5 (Media Cybernetics). The antibodies used in this study are shown in Supplemental Table 2.

Culture of primary cardiomyocytes and the autophagic flux assay

For the isolation of neonatal rat cardiac myocytes (NRCMs), hearts obtained from 1-2-day-old SD rats were sliced into $>12$ pieces and dissociated with trypsin $(0.25 \%)$. The isolated cardiomyocytes were cultured in DMEM/F12 plus with $10 \%$ FBS for $24 \mathrm{~h}$. Then, the FBS-containing medium was changed with DMEM/F12 without FBS (Xie et al. 2019).

The autophagic flux assay was performed to evaluate mRFP-LC3 and GFP-LC3 puncta localization. GFP and RFP tags were fused to the C-termini of the autophagosome marker, LC3 (mRFP-GFPLC3). This assay allows the degradation of acidsensitive GFP in autolysosomes while the stability of the acid-insensitive RFP is maintained. The yellow puncta indicate autophagosome, while the red puncta represent autolysosomes. The autophagic flux was given by the ratio of autophagosomes (yellow puncta) to autolysosomes (LC3B red puncta) (Xie et al. 2019).

First, NRCMs were infected with the adenovirus expressing mRFP-GFP-LC3 along with siRNAATGL (Ad-siRNA-ATGL) without GFP, or the scramble siRNA-control $(\mathrm{MOI}=50)$ for $24 \mathrm{~h}$. Then, they were pretreated with rapamycin (RAPA, $20 \mathrm{nM}$, mTOR inhibitor), 3-MA (5 mM, autophagy inhibitor), VO-OHpic (50 nM, PTEN inhibitor), or epoxomicin (100 $\mathrm{nM}$, proteasome inhibitor) and then stimulated with $100 \mathrm{nM}$ Ang II for $48 \mathrm{~h}$ (Bu et al. 2020; Chen et al. 2019; Meng et al. 1999; Yan et al. 2019). LC3positive puncta were examined by confocal microscopy (Leica STELLARIS 5 Microsystems, Wetzlar, Germany). Then, counts of autophagosomes (yellow) and autolysosomes (red) in each condition (20-30 cells per group) were analyzed with the ImageJ (NIH, Bethesda, MD, USA).

\section{Evaluation of the proteasome activities}

The fluorogenic peptide substrates, including Z-LLEAMC $(45 \mu \mathrm{mol} / \mathrm{L})$, Ac-RLRAMC $(40 \mu \mathrm{mol} / \mathrm{L})$, and Suc-LLVY-AMC $(18 \mu \mathrm{mol} / \mathrm{L})$ were used to measure cardiac proteasome caspase-like, trypsin-like and chymotrypsin-like activities, respectively (Chen et al. 2019; Xie et al. 2019; Zou et al. 2019). Briefly, $20 \mu \mathrm{g}$ of tissue proteins was added to $100 \mu \mathrm{L}$ of $50 \mathrm{mM}$ HEPES (pH 7.5, $5 \mathrm{mmol} / \mathrm{L} \mathrm{MgCl} 2,20 \mathrm{mmol} / \mathrm{L} \mathrm{KCl}$, and $1 \mathrm{mmol} / \mathrm{L}$ DTT), after which they were incubated with fluorogenic peptide substrates in the presence or absence of the proteasome inhibitors epoxomicin $(5 \mu \mathrm{mol} / \mathrm{L})$ or MG132 $(20 \mu \mathrm{mol} / \mathrm{L})$ at $37^{\circ} \mathrm{C}$ for $10 \mathrm{~min}$. Fluorescence intensities were assessed at the excitation and emission wavelengths of $380 \mathrm{~nm}$ and $460 \mathrm{~nm}$.

\section{Statistical analysis}

All results are shown as the mean \pm SEM. Comparisons of between groups means were performed with the unpaired two-tailed Student's $t$ test, while comparisons of means among groups were conducted with one-way ANOVA followed by Newman-Keuls for multiple comparisons. GraphPad Prism 5.0. was set as the threshold for statistical significance. 
a

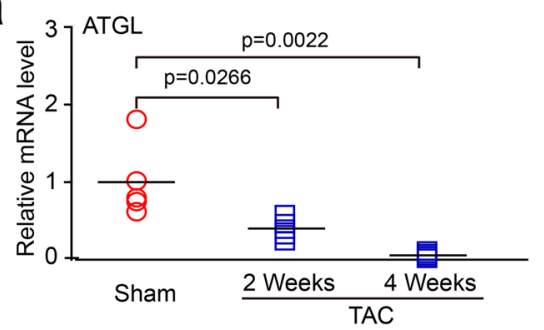

b
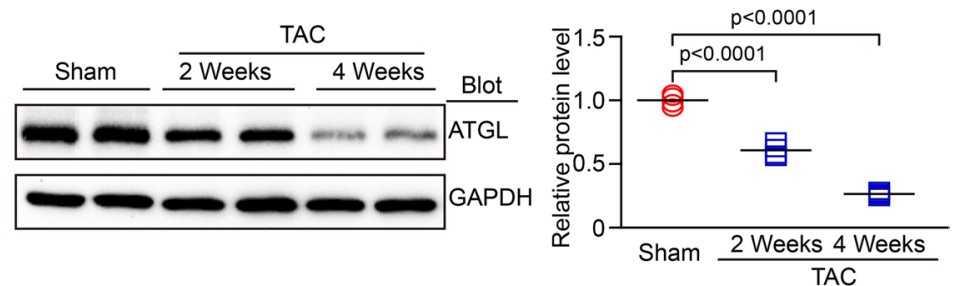

C
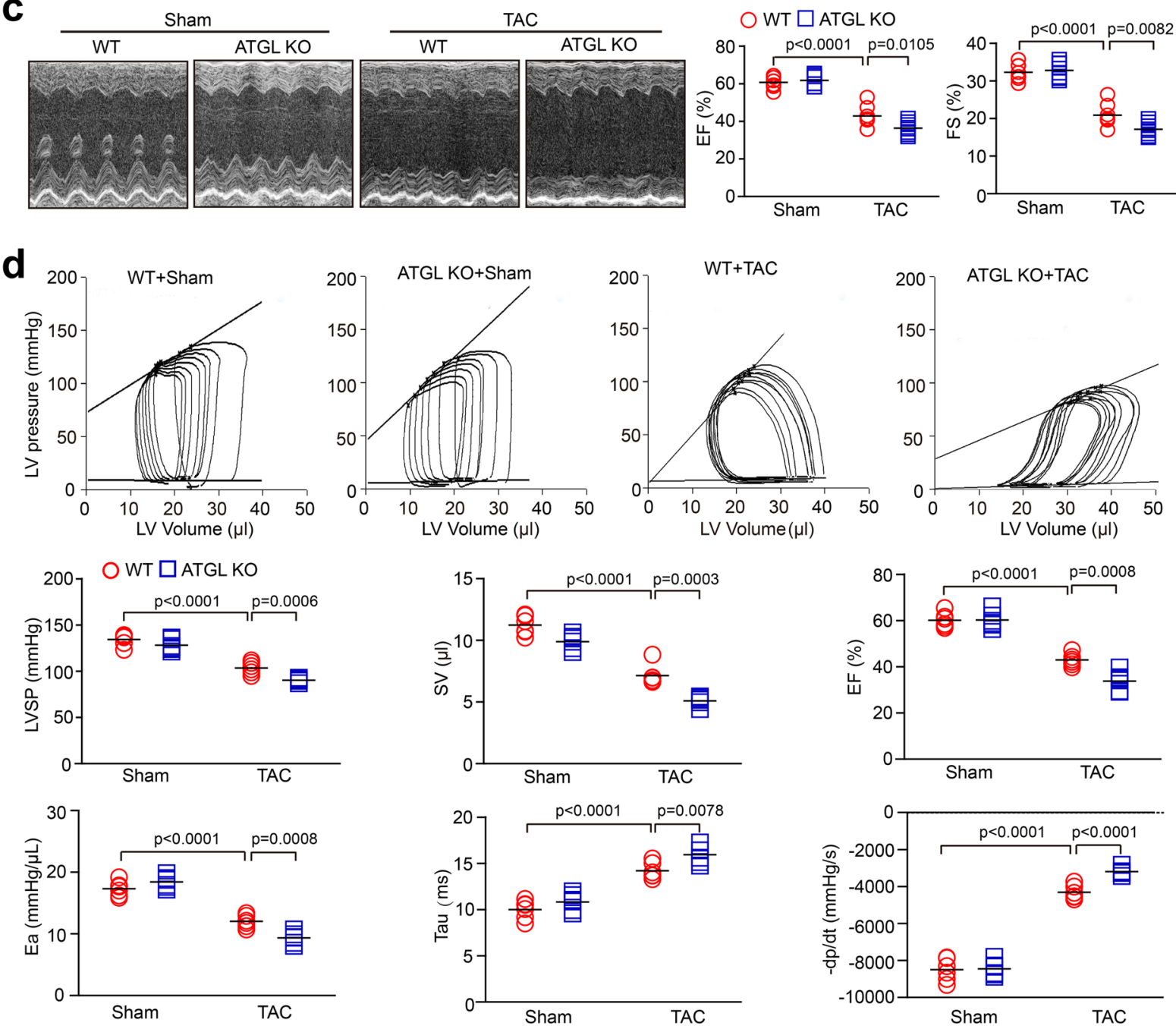

Fig. 1 Ablation of ATGL aggravates pressure overloadinduced cardiac dysfunction. Wild-type (WT) and ATGL knockout (KO) mice were subjected to sham or transverse aortic constriction (TAC) operation for 2-4 weeks. a, qPCR analysis of the ATGL mRNA level in WT hearts at weeks 2 and 4 of TAC operation $(n=5)$. b. Immunoblotting analysis of the ATGL protein level in WT heart $(n=4)$. c, Representative echocardiographic measurement of left ventricular (LV) chamber (left), and the calculation of LV ejection fraction (EF\%) and fractional shortening (FS\%) after 4 weeks of TAC operation (right, $n=8$ ). d, Pressure-volume analysis of systolic and diastolic function. Representative LV pressure-volume loops in each group (top). Summary data on systolic function and diastolic function (bottom, $n=6$ ). LVSP, LV systolic pressure; $\mathrm{SV}$, stroke volume; EF, ejection fraction; Ea, arterial elastance; Tau, relaxation time constant; -dP/dt, maximal rate of pressure decline (diastolic indexes). Data are presented as mean \pm SEM, and $\mathrm{n}$ represents number of animals 
a
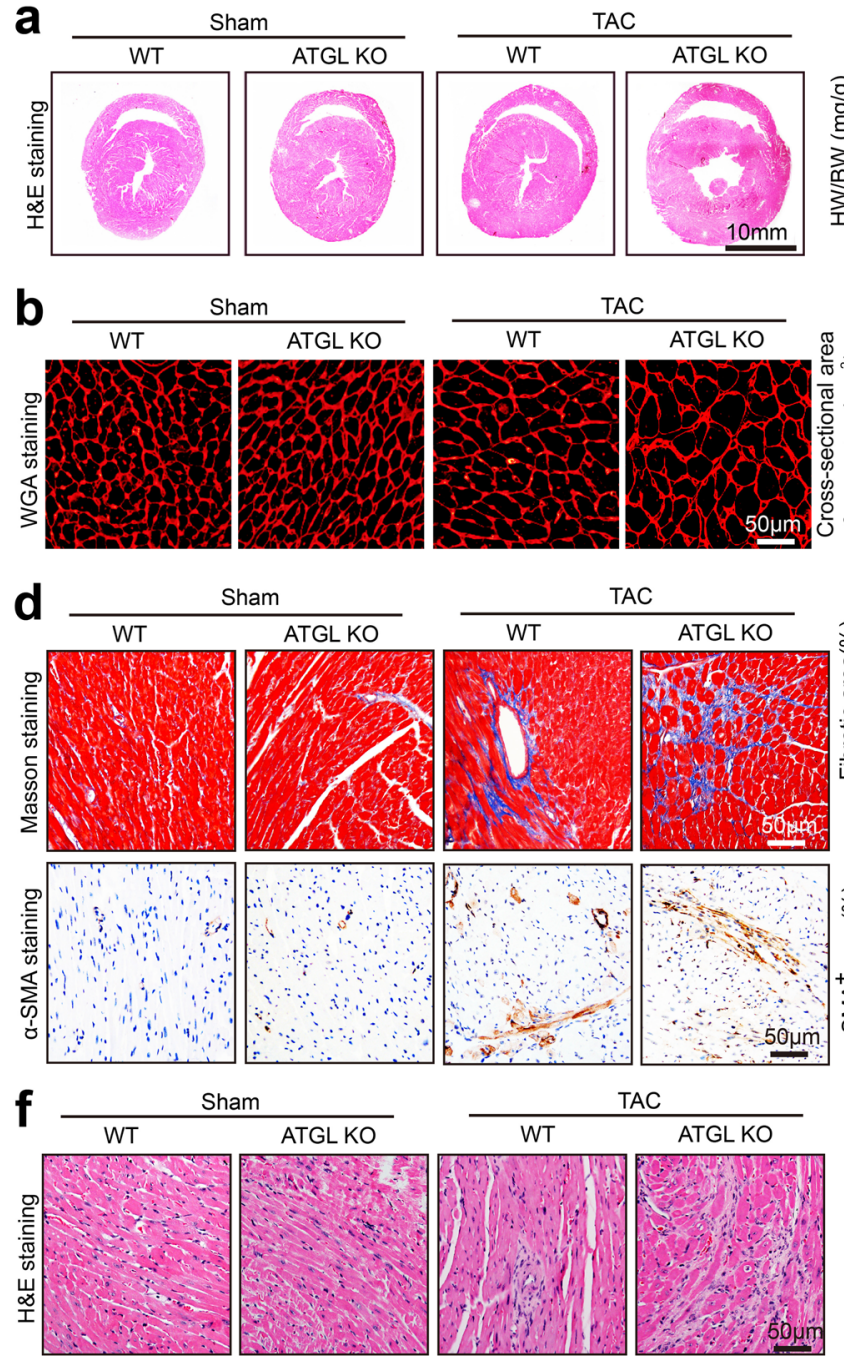

TAC
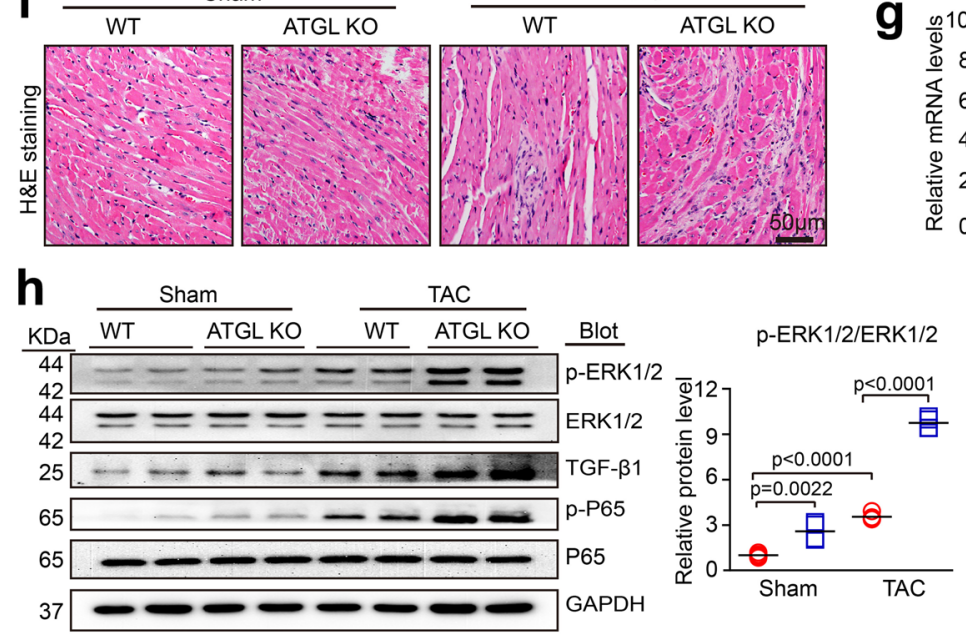

\section{Results}

ATGL KO aggravates TAC surgery-induced myocardial dysfunction

To assess the role of ATGL in the regulation of myocardial hypertrophic remodeling and function, WT g
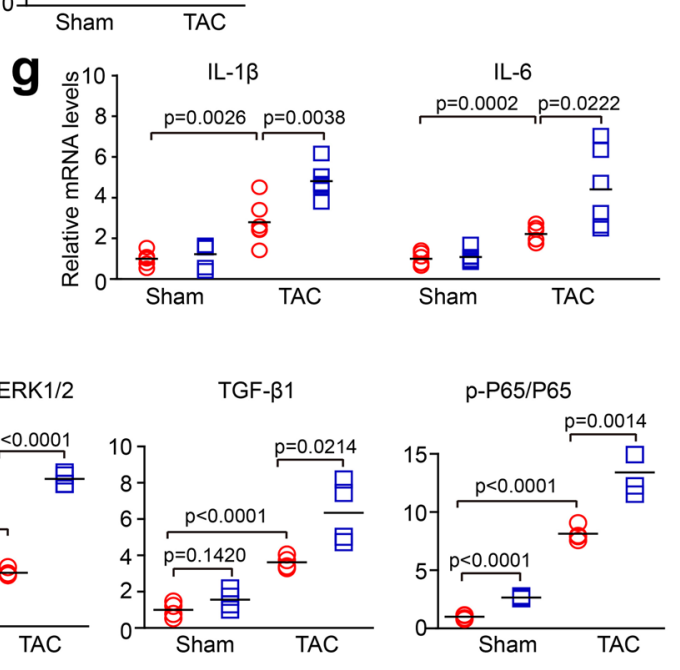

O WT $\square$ ATGL KO
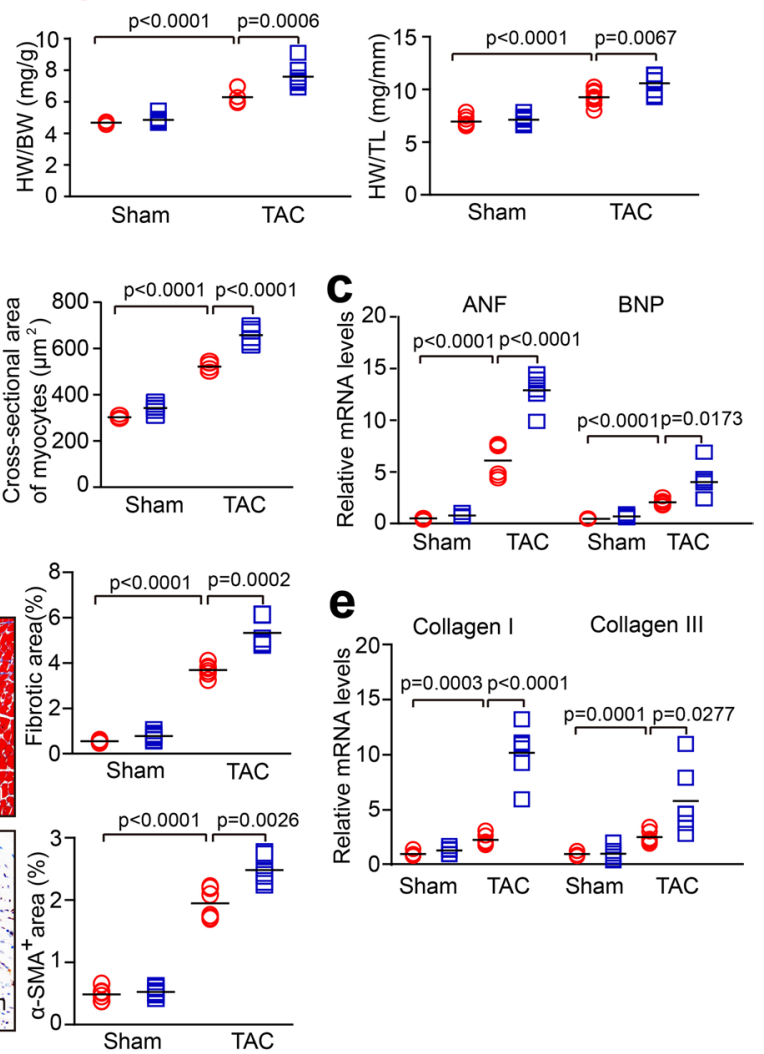

and ATGL KO mice were subjected to TAC surgery for 2 to 4 weeks to establish in vivo model of pressure overload-induced hypertrophic remodeling and dysfunction. At 1 week post TAC surgery, hemodynamic measurements revealed that the transaortic pressure gradient was significantly elevated in TAC-operated ATGL KO or WT mice, compared to sham group 
4Fig. 2 Knockout of ATGL promotes pressure overloadinduced cardiac hypertrophic remodeling. a, WT and ATGL KO mice were subjected to sham or TAC operation for 4 weeks. Representative images of heart sections (left). Scale bar: $10 \mathrm{~mm}$. The ratios of heart weight to body weight (HW/ $\mathrm{BW}$ ) and heart weight tibial length (HW/TL) (right, $n=8$ ). b, Representative images of TRITC-labeled wheat germ agglutinin (WGA) staining to measure cardiac myocyte size (left). Quantification of cross-sectional area of myocytes (right, $n=6$, 150-200 cells counted per sample). c, qPCR analyses of ANF and BNP mRNA levels in the heart $(n=6)$. d, Representative images of Masson's trichrome and immunohistochemical staining to examine fibrosis (top) and the number of myofibroblasts (bottom), respectively. Quantification of fibrotic area (\%) and the percentage of $\alpha$-SMA-positive myofibroblasts (\%) (right, $n=6$ ). e, qPCR analyses of Collagen I and Collagen III mRNA levels $(n=6)$. f, Representative images of H\&E staining of heart sections. Scale bar: $50 \mu \mathrm{m}$. g, qPCR analyses of IL-1 $\beta$ and IL- 6 mRNA levels $(n=6)$. h, Immunoblotting analyses of p-ERK1/2, ERK1/2, TGF- $\beta 1$, p-P65, P65, and GAPDH in the heart (left). Quantification of the relative protein levels (right, $n=4$ ). Data are presented as mean \pm SEM, and $n$ represents number of animals

mice, but was comparable between 2 groups following sham or TAC surgery (Supplementary Fig. 1a). Meanwhile, compared to baseline control, ejection fraction percentage $(\mathrm{EF} \%)$ and fractional shortening percentage (FS\%), which are indices for cardiac contractile function, were markedly elevated in WT mice until week 2, and then decreased at weeks 3-4 following TAC surgery. However, in ATGL KO mice, cardiac dysfunction time-dependently decreased and peaked at week 4 (Supplementary Fig. 1b). Moreover, ATGL mRNA and protein levels in TAC-operated hearts were time-dependently suppressed compared to sham-operated controls (Fig. 1a-b). Echocardiography revealed that relative to the sham group, TAC-treated WT mice exhibited markedly reduced of cardiac contractile functions (decreased FS\% and $\mathrm{EF} \%$ ), and that these parameters were further reduced in ATGL KO mice (Fig. 1c). Then, we assessed the effects of ATGL KO on cardiac functions using invasive pressure-volume analysis. After 4 weeks of TAC surgery, there was no sudden death or cardiac arrhythmia in ATGL KO or WT mice. However, TAC-operated WT mice displayed a rightward shift of end-systolic pressure-volume $(\mathrm{P}-\mathrm{V})$ relation accompanied by reduced left ventricle systolic pressure (LVSP), stroke volume (SV), EF, as well as arterial elastance (Ea), and increased Tau and maximal rate of pressure decrease $(-\mathrm{dP} / \mathrm{dt})$. These effects were more pronounced in ATGL KO mice (Fig. 1d; Supplemental
Table 3), but were not significantly different between ATGL KO and WT animals after sham operation (Fig. 1c-d). These findings imply that ATGL KO promotes pressure overload-induced cardiac dysfunction.

ATGL deficiency aggravated TAC-induced cardiac inflammation, fibrosis, and hypertrophy

Next, we determined if ATGL KO affects cardiac remodeling. Relative to sham group, TAC for 4 weeks in WT mice markedly accelerated myocardial hypertrophy, as shown by higher ratios of heart weight/ body weight (HW/BW) and heart weight/tibia length (HW/TL), left ventricular (LV) wall thickness, cardiac myocyte size, and the mRNA levels of ANF and BNP. These effects were more enhanced in TACtreated ATGL KO mice (Fig. 2a-c). Furthermore, TAC-induced elevations in LV fibrosis and inflammatory responses were observed in WT mice, as indicated by increased fibrotic areas, staining density of $\alpha$-SMA, inflammatory cell infiltrations as well as the mRNA expressions of proinflammatory cytokines (IL-1 $\beta$ and IL-6) and fibrotic markers (Collagen I and III) in TAC-operated ATGL KO hearts, relative to TAC-operated WT hearts (Fig. 2d-g). In addition, TAC surgery highly upregulated ERK1/2 and P65 phosphorylation levels and TGF- $\beta 1$ protein level in WT hearts, and this upregulation was even higher in TAC-operated ATGL KO hearts (Fig. 2h). There were similar changes in these parameters for myocardial hypertrophy, fibrosis, inflammation between two groups following sham surgery (Fig. 2a-h).

ATGL KO accelerated TAC-induced oxidative stress and cardiomyocyte apoptosis in mice

We then determined if ATGL KO affects superoxide production and apoptosis, the main causes of myocardial dysfunction. The dihydroethidium (DHE) staining and TUNEL assays indicated that DHE intensities and the percentage of TUNEL-positive cells were significantly higher in TAC-operated WT hearts than in sham-operated controls, and these effects were markedly accelerated in TAC-treated ATGL KO hearts (Fig. 3a-b). Accordingly, the levels of apoptosis-regulating proteins, such as Bax to Bcl-2 ratio and cleaved caspase-3 were also highly enhanced, but levels of p-AMPK $\alpha$ (a key regulator of mitochondrial ATP generation) and ATP production 
were significantly suppressed in ATGL KO hearts relative to WT hearts following sham or TAC surgery (Fig. 3c-d). However, ATP production levels in ATGL KO hearts and WT hearts were comparable after sham surgery (Fig. 3e).

ATGL deficiency enhanced TAC-induced inhibition of autophagy

To evaluate the potential mechanisms through which ATGL KO aggravated TAC-induced cardiac dysfunction, we first analyzed the effects of ATGL KO on myocardial lipid deposition. Red oil staining revealed that there were no significant changes in the myocardial lipid levels, including TG content in ATGL KO mice relative to WT controls following sham or TAC surgery (Supplementary Fig. 2ab). Moreover, the levels of TG, TC, LDL-C, and HDL-C in plasma of ATGL KO and WT mice were comparable (Supplementary Fig. 2c). These results indicate that ATGL KO did not affect cardiac lipid metabolism.

Given the role of ATGL in autophagy activation during hepatic lipid metabolism (Martinez-Lopez et al. 2016; Sathyanarayan et al. 2017), we therefore examined autophagy signaling mediators, that are crucial for cardiomyocyte apoptosis and cardiac remodeling. We observed that TAC significantly inactivated autophagy, as indicated by increased protein levels of p-mTOR, p-ULK1, Atg13, and p62 as well as suppressed Atg5 levels and LC3II/I ratios in WT hearts. This inhibitory effect was further enhanced in TAC-treated ATGL KO hearts (Fig. 4a). Interestingly, the protein levels of p-ULK1, Atg13, and p62 were higher while Atg5 level was lower in ATGL KO hearts than in WT hearts after sham surgery (Fig. 4a).

To confirm the effects of ATGL KO on autophagy, we assessed autophagic flux in neonatal rat cardiomyocytes (NRCMs) using tandem fluorescent mRFP-GFP-LC3 along with Ad-siRNA control or Ad-siRNA-ATGL. After $48 \mathrm{~h}$ of Ang II treatment, both the number of both autolysosomes (red) and autophagosomes (yellow) were markedly reduced but cardiomyocyte sizes were enhanced in AdsiRNA-ATGL-infected NRCMs compared with AdsiRNA-controls (Fig. 4b-c). Similarly, the number of autophagosomes (yellow) was also lower in AdsiRNA-ATGL cells after saline treatment (Fig. 4b), indicating that ATGL KO reduced autophagic flux.
To determine if autophagy is a downstream target of ATGL, WT or ATGL KO mice were treated with rapamycin (RAPA, the mTOR inhibitor) to activate autophagy or 3-MA to inhibit autophagy after which they were continuously subjected to TAC for 4 weeks. In WT mice, RAPA significantly activated autophagy, as indicated by suppressed levels of p-mTOR, p-ULK1 and elevated LC3II/I ratios compared with vehicle-treated controls. In contrast, 3-MA markedly inhibited autophagic activation (Fig. 4d). Similarly, in TAC-operated ATGL KO mice, the effect of RAPA or 3-MA on autophagy was further agrravated compared with vehicle-treated controls (Fig. 4d).

Then, we confirmed the influence of ATGL KO on autophagy by assessing autophagic flux in NRCMs via infections by the Ad-siRNA-control or AdsiRNA-ATGL in the presence or absence of RAPA or 3-MA. Compared to vehicle-treated controls, RAPA markedly elevated autophagic flux, as shown by increased numbers of autolysosomes (red) and autophagosomes (yellow) in both Ad-siRNA-controland Ad-siRNA-ATGL-infected NRCMs. Treatment with 3-MA reversed this effect following Ang II treatment (Fig. 4e). Together, these results suggest that ATGL KO inhibits autophagy.

Autophagy is involved in ATGL KO-mediated cardiac dysfunction and hypertrophic remodeling

To determine whether autophagy is involved in ATGL KO-mediated dysfunction and cardiac remodeling, WT or ATGL KO mice were treated with RAPA or 3-MA and then continuously subjected to TAC for 4 weeks. In WT mice, TAC-induced cardiac dysfunction (decreased FS\%) was remarkably restored by RAPA, but aggravated by 3-MA (Fig. 5a). The effects of RAPA or 3-MA on TAC-induced suppression of cardiac functions (Alterations of $\mathrm{P}-\mathrm{V}$ loop, LVSP, $\mathrm{SV}, \mathrm{EF}, \mathrm{Ea}$, Tau, and - dP/dt) were confirmed by invasive pressure-volume analysis (Fig. 5b; Supplemental Table 4). Accordingly, TAC-triggered cardiac hypertrophy (reduced heart sized, LV wall thickness, HW to TL ratio, and myocyte area) (Fig. 5c-d), interstitial fibrosis (Fig. 5e), infiltration of Mac- $2^{+}$macrophages (Fig. 5f), superoxide production (Fig. $5 \mathrm{~g}$ ), and the percentage of $\mathrm{TUNEL}^{+}$myocytes (Fig. 5h) were significantly attenuated in RAPA-treated hearts relative to vehicle-treated hearts (Fig. 5c-h). However, these effects were enhanced in 3-MA-treated WT mice 
a

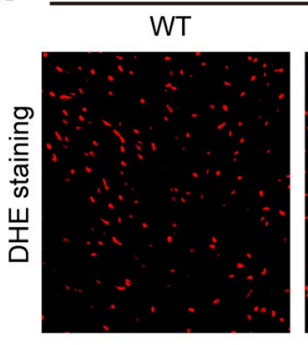

b
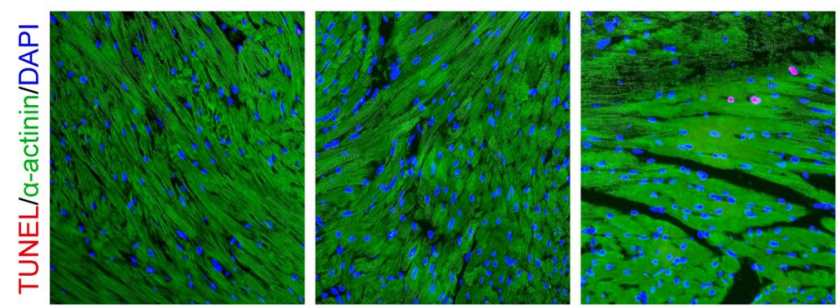

C
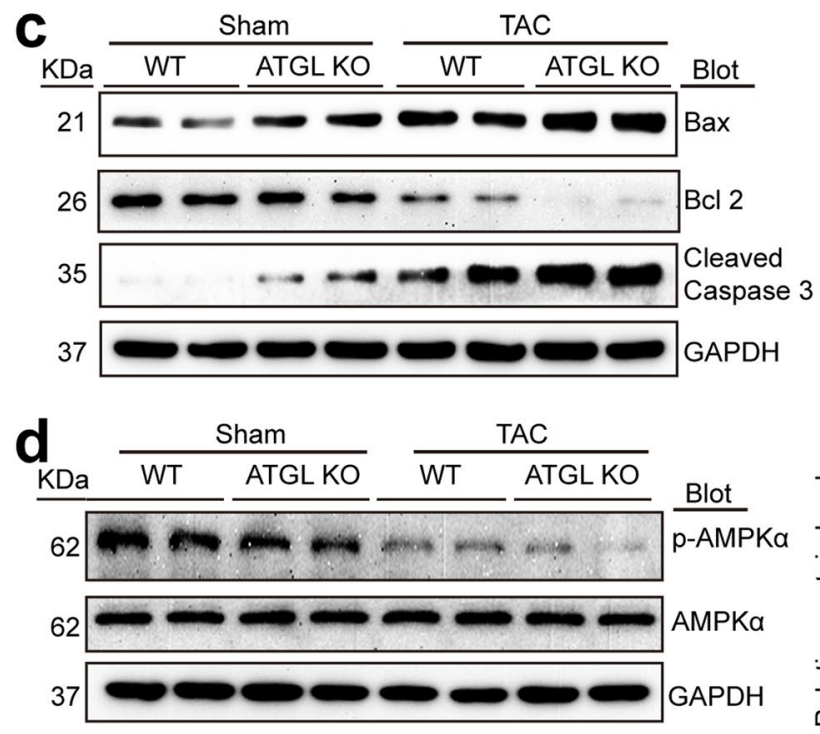

Fig. 3 Deficiency of ATGL accelerates pressure overloadinduced cardiac oxidative stress and apoptosis. a, WT and ATGL KO mice were subjected to sham or TAC operation for 4 weeks. Representative images of dihydroethidium (DHE) staining of the heart sections (left). Quantification of the relative superoxide production (right, $n=6$ ). Scale bar: $50 \mu \mathrm{m}$. b, Representative images of TUNEL (red), $\alpha$-actinin (green) and DAPI (blue) staining of the heart sections (left), and quantification of TUNEL-positive nuclei (right, $n=6$ ). Scale

(Fig. 5a-h). Moreover, in ATGL KO mice, the effects of RAPA or 3-MA on TAC-triggered cardiac hypertrophy and dysfunction were comparable to WT mice

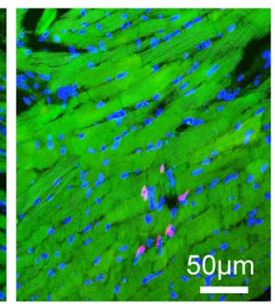

TAC

ATGL KO
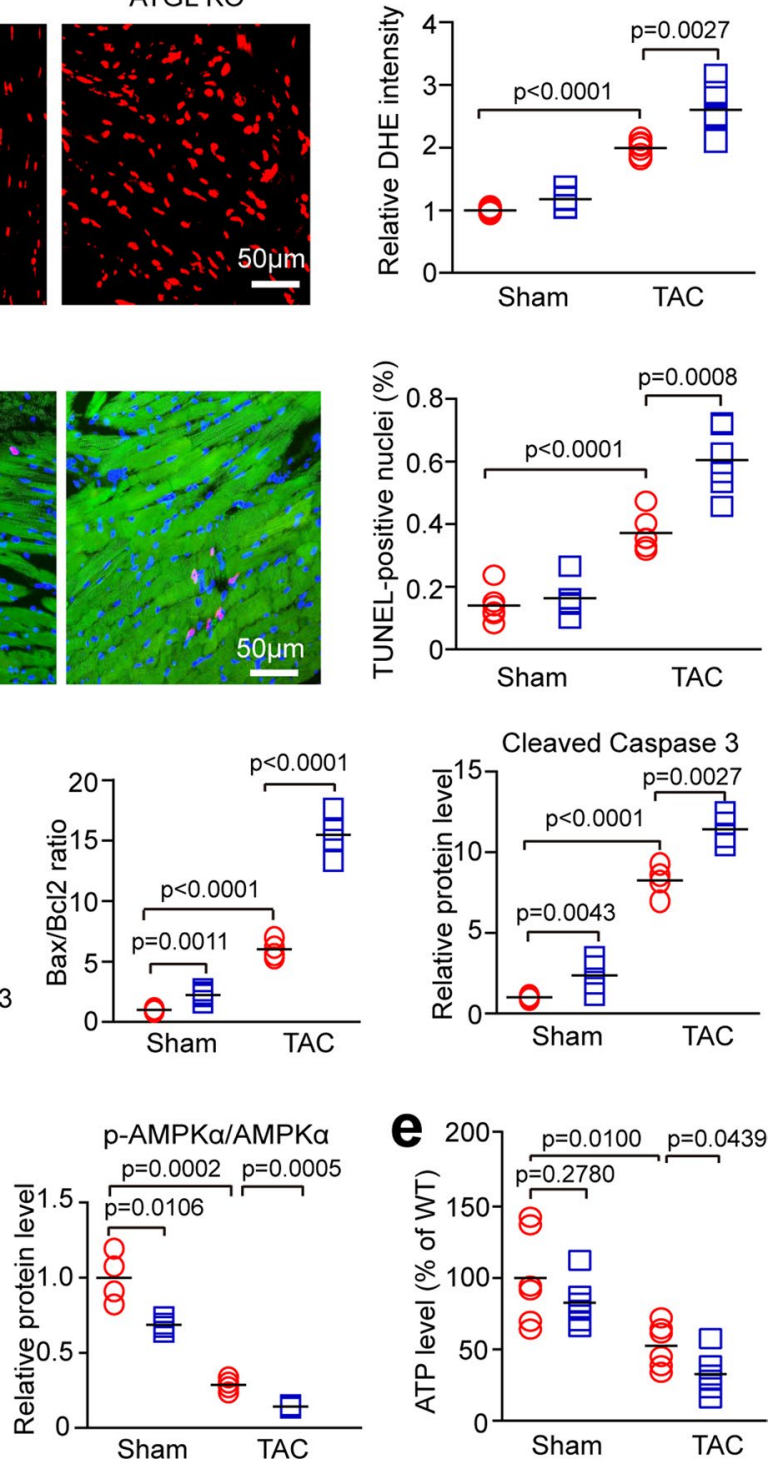

bar: $50 \mu \mathrm{m}$. c, Representative immunoblotting of Bax, Bcl-2, cleaved capsase-3 and GAPDH in the heart (left). Quantification of the relative protein levels (right, $n=4$ ). d, Representative immunoblotting of p-AMPK $\alpha$, AMPK $\alpha$ and GAPDH in the heart (left). Quantification of the relative protein levels (right, $n=4$ ). e, Measurement of ATP level in the heart tissues. Data are presented as mean $\pm S E M$, and $n$ represents number of animals

but were further enhanced (Fig. 5a-h; Supplemental Table 4). Therefore, autophagy participated in TACinduced cardiac remodeling in ATGL KO mice. 

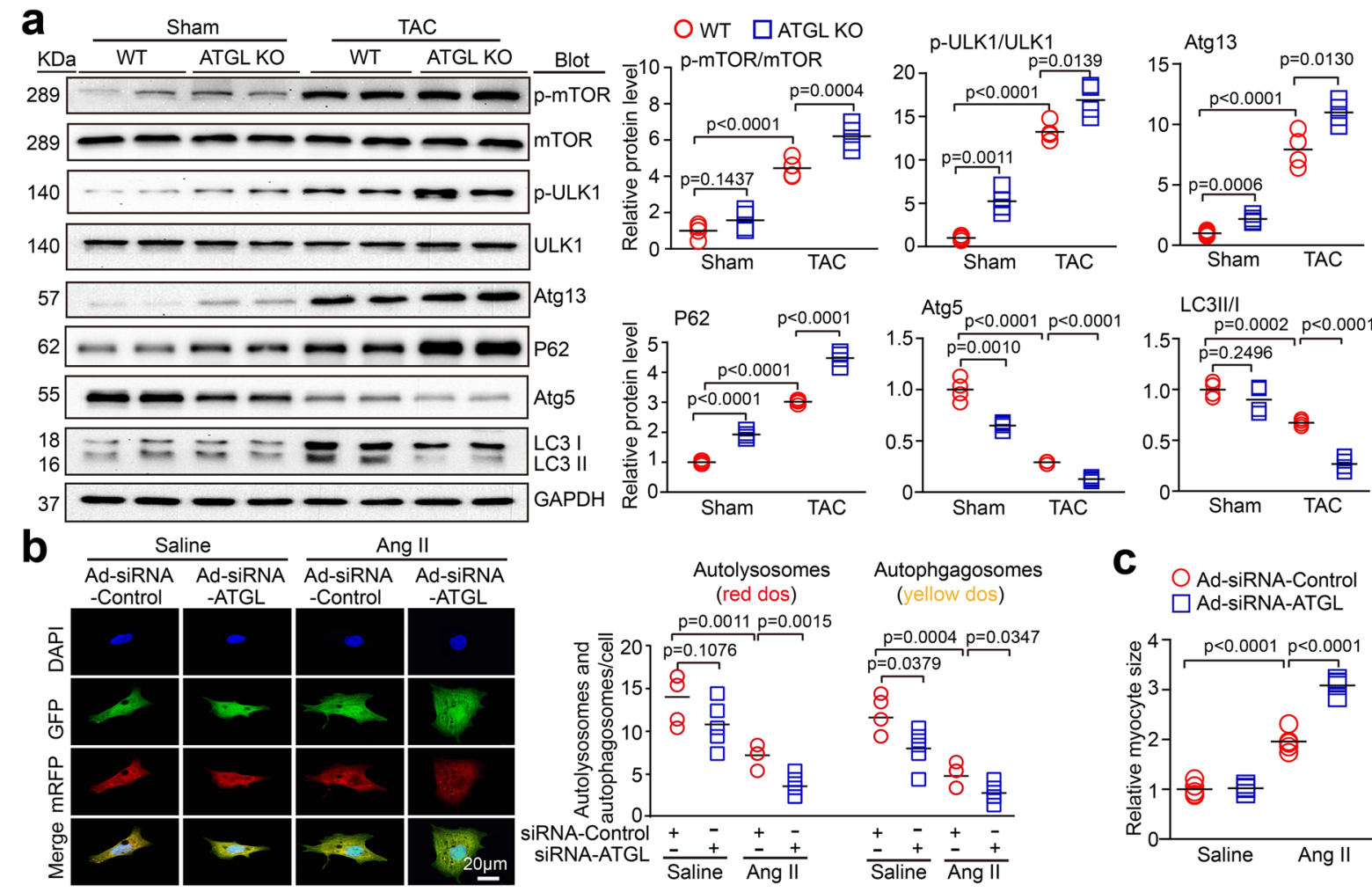

LC3II/I

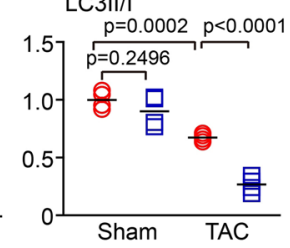

d
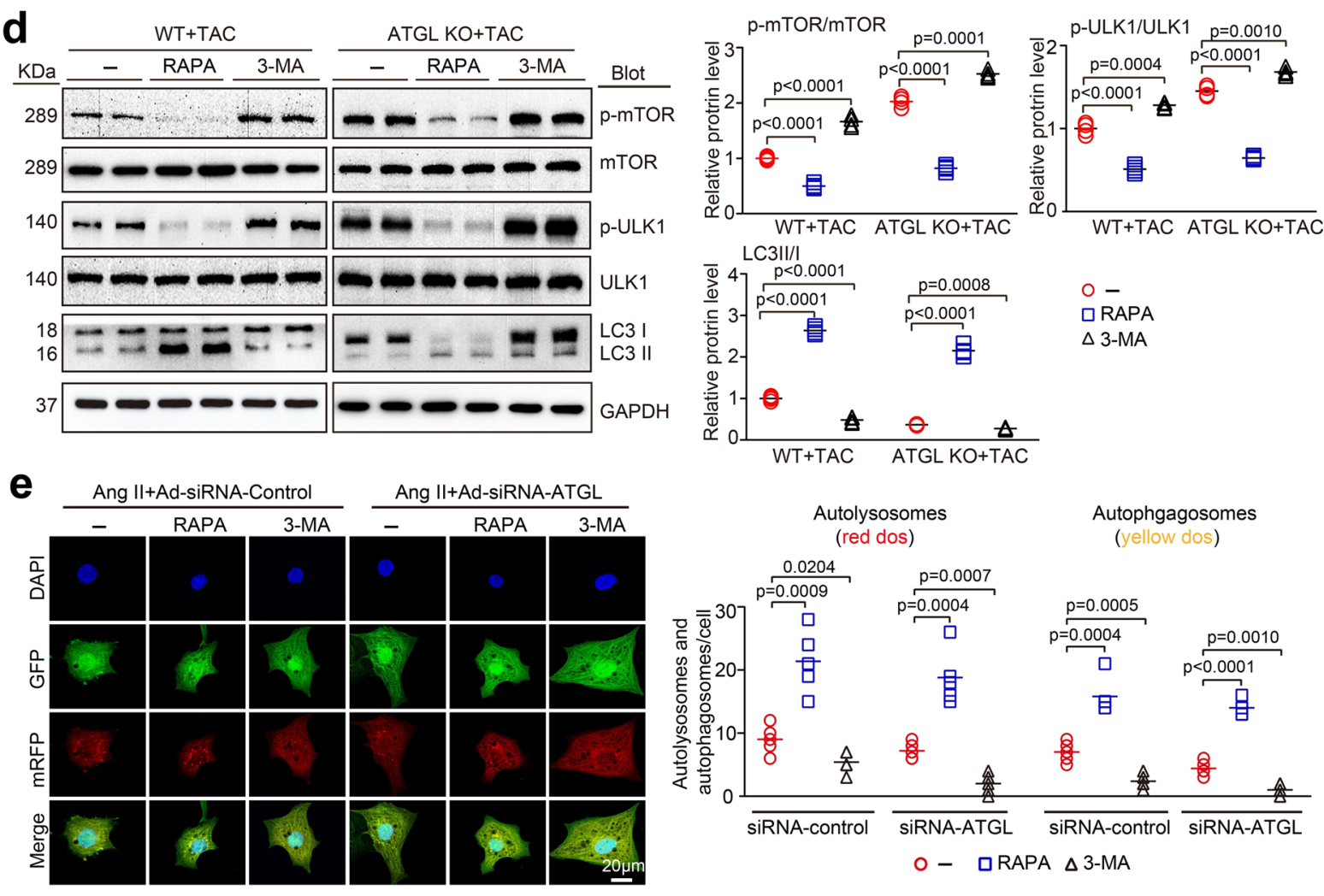
\Fig. 4 ATGL knockout enhances pressure overload-induced inhibition of autophagic signaling. a, WT and ATGL KO mice were subjected to sham or TAC operation for 4 weeks. Representative immunoblotting of p-mTOR, mTOR, p-ULK1, ULK1, Atg13, p62, LC3, Atg5, and GAPDH in the heart (left). Quantification of the relative protein levels (right, $n=4$ ). b, Neonatal rat cardiomyocytes (NRCMs) were infected with adenovirus containing mRFP-GFP-LC3 together with AdsiRNA-control or Ad-siRNA-ATGL and then treated with Ang II (100 nM) for $48 \mathrm{~h}$. Immunofluorescence images (right) and the quantification of autophagosomes (yellow) and autolysosomes (red) in each condition (right, 15-20 cells per group) ( $n=5$ independent experiments). Scale bar: $20 \mu \mathrm{m}$. c, The quantification of cardiomyocyte size in each condition (right, $15-20$ cells per group) ( $n=5$ independent experiments). Scale bar: $20 \mu \mathrm{m}$. d, WT and ATGL KO mice were subjected to TAC and co-treated with rapamycin (RAPA, $4 \mathrm{mg} / \mathrm{kg}$ daily) or 3-MA (10 mg/kg daily) for 4 weeks. Representative immunoblotting of p-mTOR, mTOR, p-ULK1, ULK1, and LC3 and GAPDH in the heart (left). Quantification of the relative protein levels (right, $n=4)$ ). e, NRCMs were infected with adenovirus containing mRFP-GFP-LC3 together with Ad-siRNAcontrol or Ad-siRNA-ATGL and then treated with Ang II $(100 \mathrm{nM})$ in the presence of RAPA $(20 \mathrm{nM})$ or 3 -MA $(5 \mathrm{mM})$ for $48 \mathrm{~h}$. Immunofluorescence images (left) and the quantification of autophagosomes (yellow) and autolysosomes (red) in each condition (right, $15-20$ cells per group) ( $n=5$ independent experiments). Scale bar: $20 \mu \mathrm{m}$. Data are presented as mean $\pm \mathrm{SEM}$, and $\mathrm{n}$ represents number of animals

ATGL KO inhibited autophagy via the proteasome-PTEN-mTOR signaling

The proteasome complex is highly induced by various hypertrophic stimuli and is crucial for regulating autophagy and PTEN stability in the hearts (Cao et al. 2019; Chen et al. 2019; Han et al. 2020; Xie et al. 2019; Xie et al. 2020; Yan et al. 2020). Therefore, we assessed the effect the of ATGL KO on the catalytic subunit expressions and activities in the hearts. Relative to sham controls, TAC surgery markedly upregulated the proteasome activities (chymotrypsinlike, trypsin-like, and caspase-like) and the protein levels of immunosubunits $(\beta 1 \mathrm{i}, \beta 2 \mathrm{i}$, and $\beta 5 \mathrm{i})$, and this effect was further enhanced in ATGL KO mice following TAC treatment (Fig. 6a-b). Furthermore, TAC-induced downregulation of PTEN (a negative regulator of $\mathrm{AKT} / \mathrm{mTOR}$ signaling) and upregulation of p-AKT in WT mice were also accelerated in ATGL KO mice (Fig. 6c), suggesting that the proteasome may be involved in the degradation of PTEN protein. Interestingly, the protein levels of Sirt1 (Sirtuin 1, an autophagy activator) (Lee et al. 2008) were not significant changed between in ATGL KO and WT hearts following sham or TAC surgery (Fig. 6c).

To define whether the proteasome regulates PTEN degradation and mTOR-mediated autophagy in vivo, we treated WT or ATGL KO mice with epoxomicin (a proteasome inhibitor) or VO-OHpic (a specific PTEN inhibitor) and then subject them to TAC continuously for 4 weeks. In both WT and ATGL KO mice, TACinduced upregulation of proteasomal caspase-like, trypsin-like, and chymotrypsin-like activities were significantly suppressed by epoxomicin but enhanced by VO-OHpic treatments (Supplementary Fig. 3). Moreover, VO-OHpic treatment markedly suppressed PTEN and LC3 II/I ratios, but enhanced p-mTOR levels, and these effects were highly reversed by epoxomicin treatment in TAC-operated WT hearts (Fig. 6d). Accordingly, after Ang II treatment, VOOHpic also markedly inhibited the autophagic flux, as indicated by decreased autolysosomes (red) and autophagosomes (yellow) in both Ad-siRNA-infected NRCMs. Conversely, epoxomicin remarkably upregulated autophagic flux (Fig. 6e). Meanwhile, the effect of VO-OHpic or epoxomicin on PTEN-mTOR-LC3 II/I signals as well as autophagic flux were further enhanced in TAC-treated ATGL KO mice (Fig. 6d, e). Collectively, these findings imply that ATGL KO inhibited autophagy by enhancing PTEN proteasomal degradation, leading to activation of autophagy in TAC-induced hypertrophic hearts.

Blockage of the proteasome activity attenuated TAC-induced cardiac remodeling in ATGL KO mice

To test whether the proteasome is involved in TACinduced cardiac dysfunction and remodeling, WT or ATGL KO mice were administrated with epoxomicin ot VO-OHpic. After 4 weeks of TAC surgery, TACinduced suppression of cardiac functions (decreased FS\%) (Fig. 7a), and enhancement of cardiac hypertrophy (increased LV wall thickness, ratios of HW/ TL, and myocyte area) (Fig. 7b-c), fibrosis (Fig. 7d), superoxide production (Fig. 7e), and myocyte apoptosis (Fig. 7f) in vehicle-treated WT hearts were further aggravated in VO-OHpic-administered ATGL KO mice (Fig. 7a-f). However, these deleterious effects were restored in epoxomicin-administered WT mice (Fig. 7a-f). Moreover, the effects of epoxomicin or VO-OHpic on cardiac dysfunction and remodeling 
a
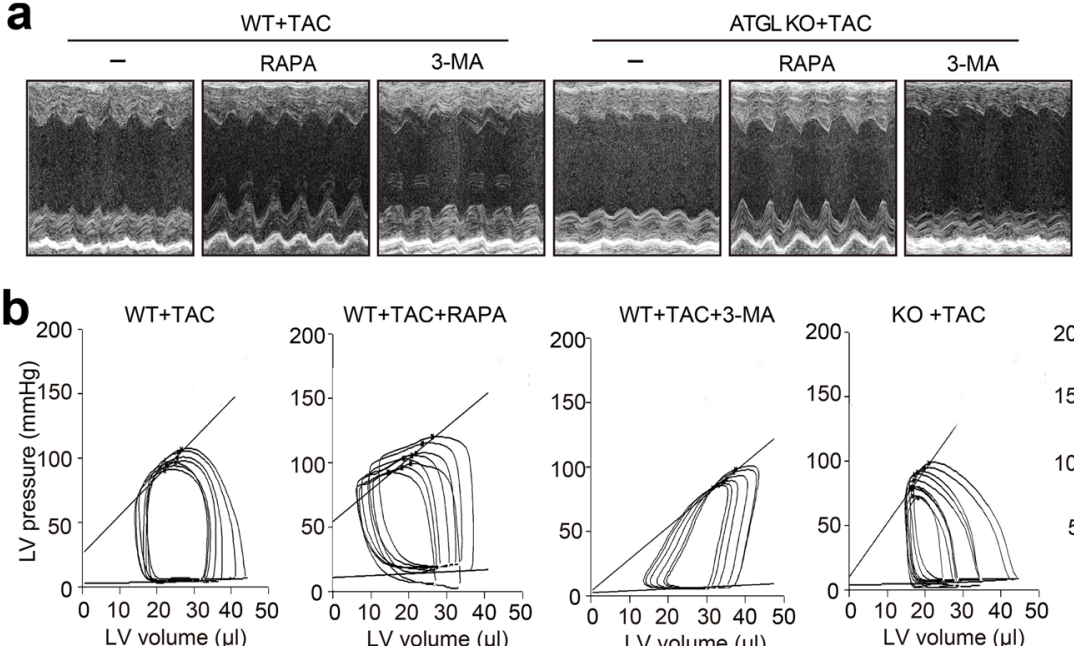

C

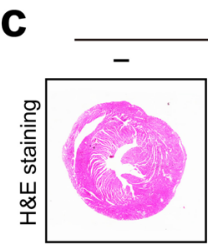

WT+TAC
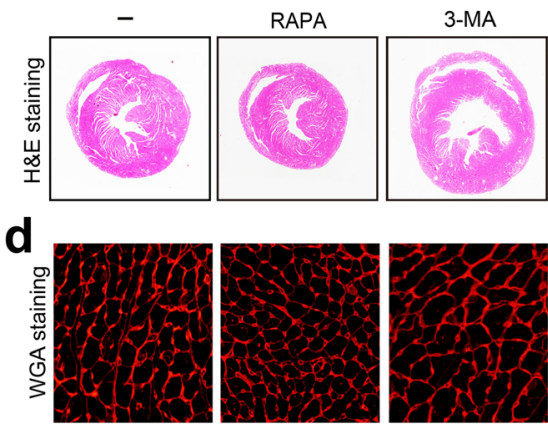

e
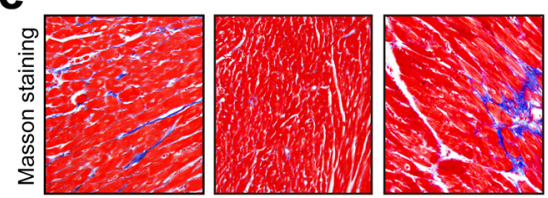

f
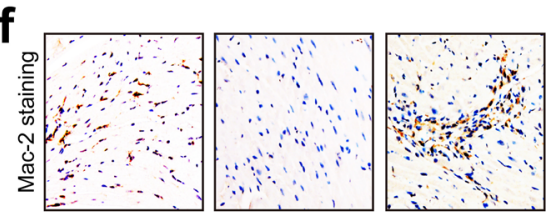

g
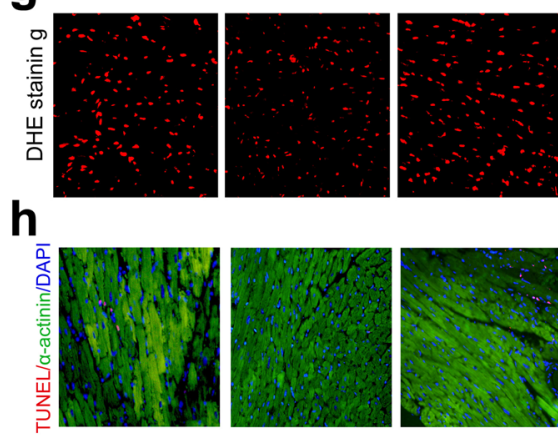
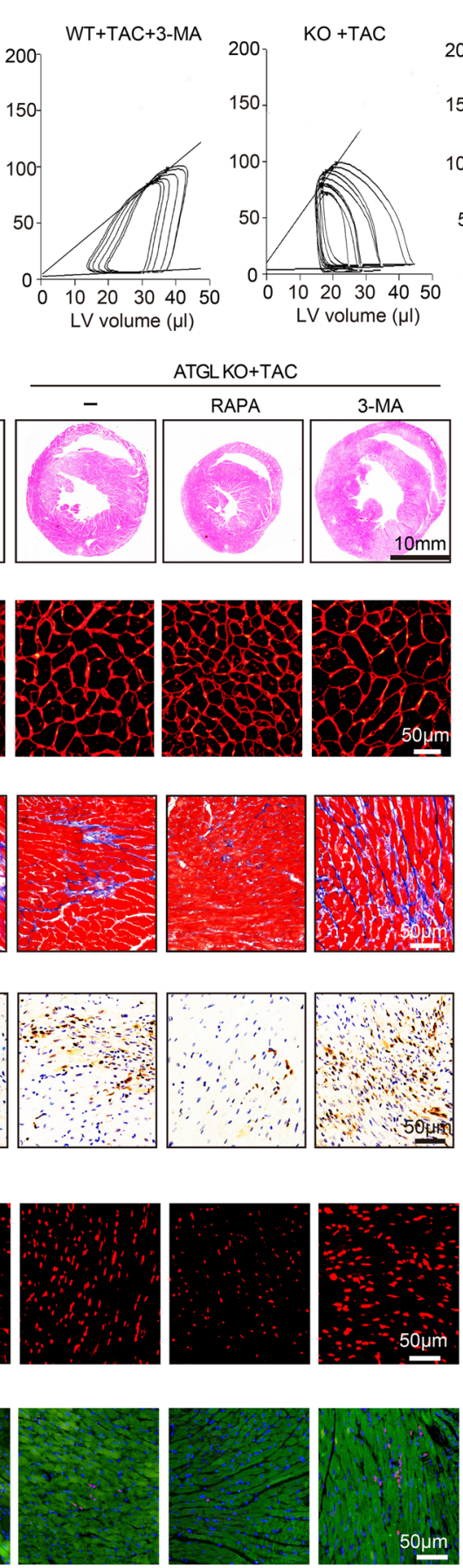

ATGLKO+TAC

O - 口RAPA $\triangle 3 M A$
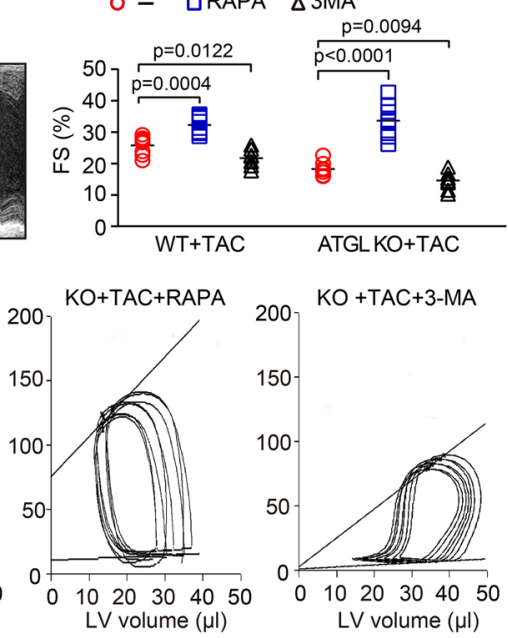

O - 口RAPA $\triangle 3 M A$

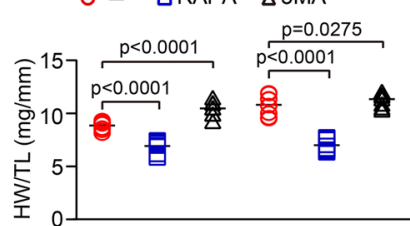

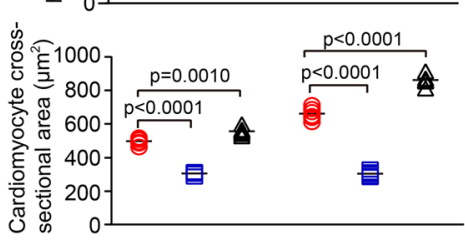

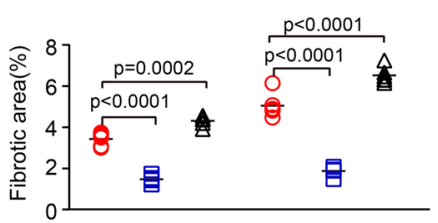

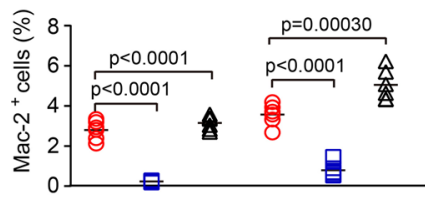

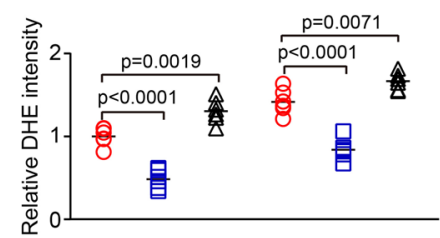

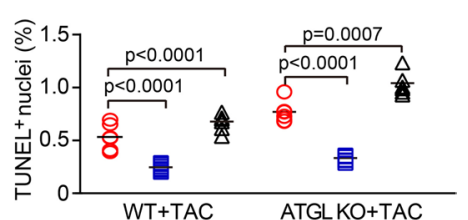


4Fig. 5 Autophagy mediates pressure overload-induced cardiac hypertrophic remodeling and dysfunction in ATGL KO mice. a, WT and ATGL KO mice were subjected to TAC and co-treated with RAPA (4 mg/kg daily) or 3-MA $(10 \mathrm{mg} /$ $\mathrm{kg}$ daily) for 4 weeks. Echocardiographic measurement of LV chamber (left), and the calculation of LV FS\% (right, $n=8$ ). b, Pressure-volume analysis of systolic and diastolic function. Representative LV P-V loop in each group. c, Representative $\mathrm{H} \& \mathrm{E}$ images of heart sections (left). Scale bar: $10 \mathrm{~mm}$. The ratio of HW/TL (right, $n=6$ ). d, TRITC-labeled WGA staining to measure cardiac myocyte size (left). The Quantification of cross-sectional area of myocytes (right, $n=6,150-200$ cells counted per sample). Scale bar: $50 \mu \mathrm{m}$. e, Masson's trichrome staining of heart sections (left), and quantification of fibrotic area (right, $n=6$ ). Scale bar: $50 \mu \mathrm{m}$. f, Immunohistochemical staining of heart sections with anti-Mac-2 antibody (left). The percentage of Mac-2-positive macrophages (right, $n=6$ ). Scale bar: $50 \mu \mathrm{m}$. g, DHE staining of heart sections (left), and quantification of the relative superoxide production (right, $n=6$ ). Scale bar: $50 \mu \mathrm{m}$. h, Staining of sections for TUNEL (red), $\alpha$-actinin (green) and DAPI (blue) (left). Quantification of the TUNEL-positive nuclei (right, $n=6$ ). Scale bar: $50 \mu \mathrm{m}$. Data are presented as mean \pm SEM, and $n$ represents number of animals

were further enhanced in TAC-operated ATGL mice (Fig. 7a-f), demonstrating that ATGL KO accelerates cardiac hypertrophic remodeling by enhancing PTEN degradation by the proteasome.

\section{Discussion}

In this study, we provide novel evidence that ATGL exerts a cardioprotective role in response to pressure overload. ATGL expression was markedly decreased in TAC-induced hypertrophic heart. ATGL KO significantly promoted TAC-induced cardiac hypertrophy and dysfunction accompanied with reduction of PTEN protein and activation of AKT-mTOR signaling as well as inhibition of autophagy. These effects were reversed by proteasome inhibitor (epoxomicin) or autophagic activator (RAPA) but accelerated by PTEN inhibitor (VO-OHpic) or autophagy inhibitor 3-MA. Mechanistically, ATGL KO upregulates proteasome subunit expression and activities, which then mediates PTEN degradation leading to activation AKT-mTOR signaling and inhibition of autophagy, thereby promoting hypertrophic remodeling and dysfunction. Overall, our results suggest that ATGL acts as a novel regulator of cardiac hypertrophic remodeling probably associated with the proteasome-PTENmTOR-autophagy pathway.
ATGL is the rate-limiting enzyme in lipolysis and is predominantly expressed in adipose tissues and in other tissues, such as heart (Kintscher et al. 2020). ATGL plays a critical role in modulating lipid metabolism and cardiac function (Foryst-Ludwig et al. 2015; Salatzki et al. 2018). Ablation of ATGL in adipose tissues or inhibition of ATGL by Atglistatin (predominantly targeting ATGL in adipose tissue) was shown to prevent cardiac damage and dysfunction by reducing galectin-3 or fatty acid secretion from adipocyte tissues (Takahara et al. 2021; Thiele et al. 2021), implying that adipocytic ATGL is involved in cardiac remodeling and dysfunctions. However, several studies have revealed that ATGL in cardiomyocytes directly regulates cardiac metabolism and mitochondrial substrate oxidation through different mechanisms (Gao et al. 2015, Haemmerle et al. 2011; Kienesberger et al. 2012). Interestingly, ATGL deficiency exhibited age-dependent upregulation of TG levels in cardiomyocytes, with starting at 6 weeks of age (Haemmerle et al. 2006). However, our results show that ATGL KO in mice at 12 weeks of age had no significant effects on cardiac TG and plasma lipid levels under basal conditions or following 4 weeks of TAC surgery (Supplementary Fig. 2). These differences in outcomes have not been elucidated; therefore, we determined if other mechanisms, such as autophagy, are involved in pressure overload-induced cardiac hypertrophic remodeling.

Autophagy is a degradative process that promotes lysosomal degradation of proteins and organelles, which is regulated by autophagy-related genes, including ULK1, Atg13, Atg5, Atg6, and Atg8/LC3 (Wang and Cui 2017). The ULK1/Atg13 complex is the most upstream mediator of autophagic induction and is phosphorylated by mTOR (Ganley et al. 2009). Autophagic dysregulation has been shown to affect cardiomyocyte apoptosis and function, leading to cardiac hypertrophy ( $\mathrm{Li}$ et al. 2016). Overexpressions of Atg6 in cardiomyocytes aggravates pressure overload-induced HF (Zhu et al. 2007). Conversely, cardiac-specific Atg5 KO enhances TCA-triggered LV hypertrophy and reduced contractile function (Nakai et al. 2007). Here, we found that ATGL KO significantly aggravated TAC-induced activation of apoptosis and inhibition of autophagy accompanied with increased $\mathrm{Bax} / \mathrm{Bcl}-2$ ratios and the protein levels of cleaved caspase-3, p-mTOR, p-ULk1, Atg13 and p62, as well as decreased Atg5 protein levels and 
a
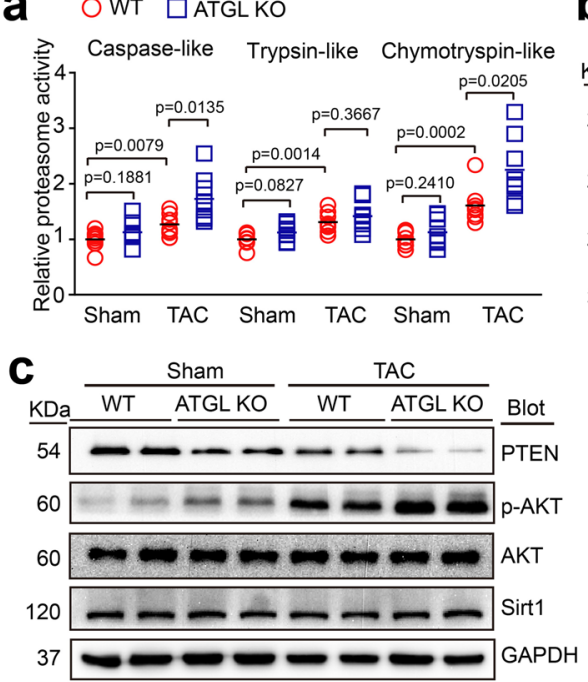

d

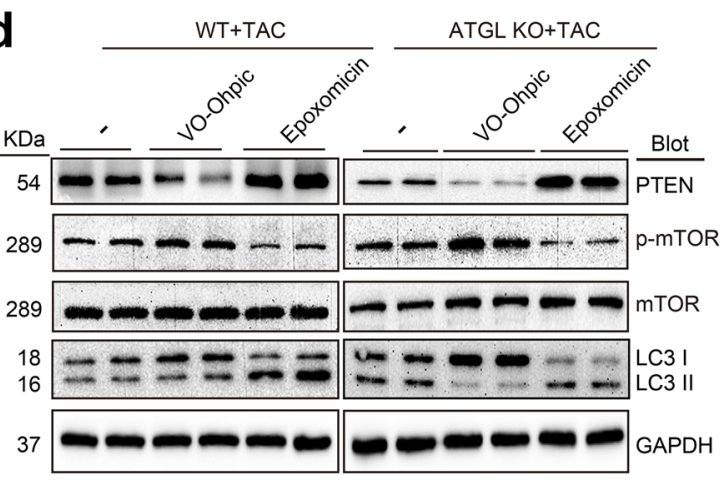

e

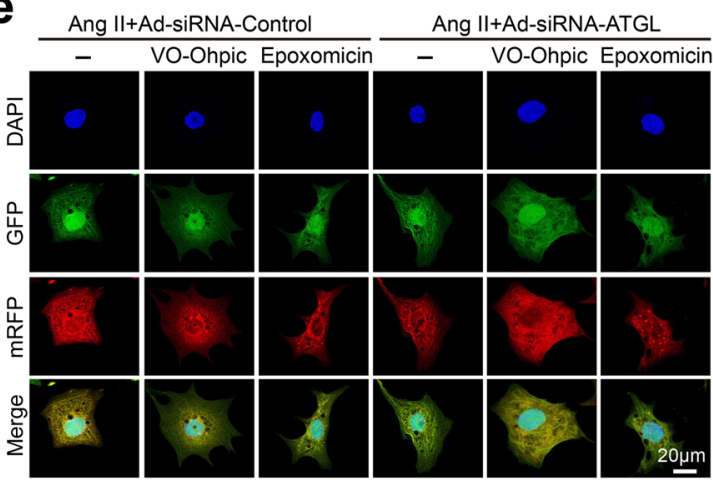

LC3II/I ratio (Fig. 3c, Fig. 4a-b). Respective activation or inhibition of autophagy in ATGL KO or WT mice using RAPA or 3-MA confirmed that ATGL KO accelerated TAC induced cardiac hypertrophy and LV dysfunction through suppressing autophagy (Fig. 4-5). However, ATGL KO also markedly
OWT $\square$ ATGLKO

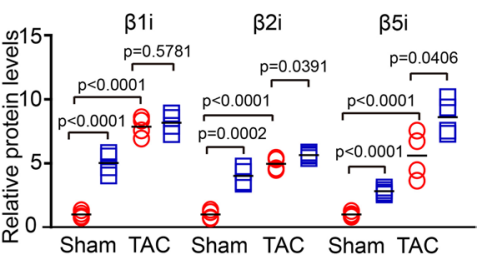

O WT $\square$ ATGL KO
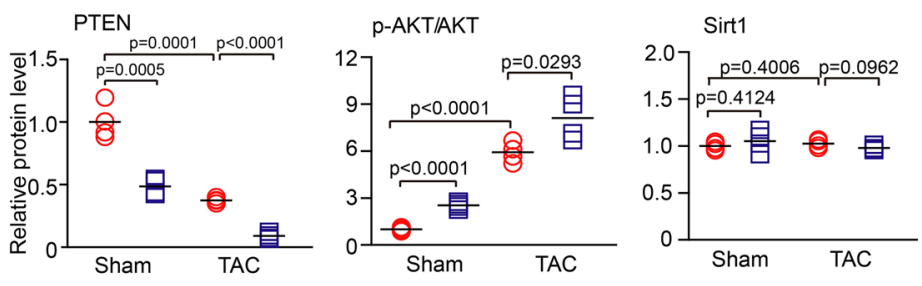

PTEN

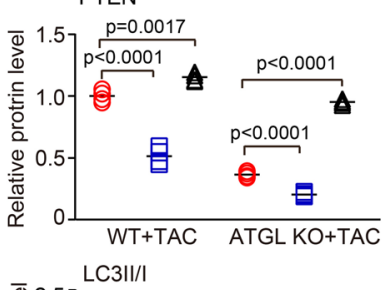

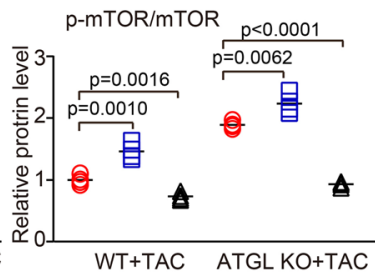

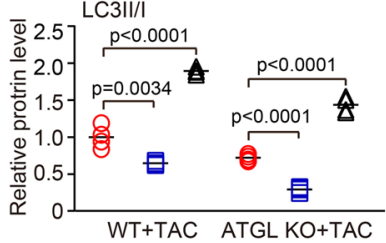

$0-$

a VO-Ohpic

$\triangle$ Epoxomicin$$
\text { 过 }
$$

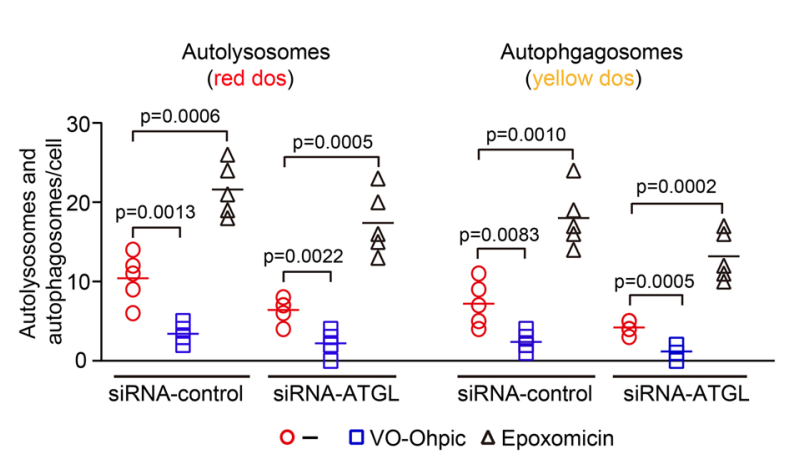

upregulated $\mathrm{Bax} / \mathrm{Bcl}-2$ ratios, cleaved caspase-3, p-ULk1, Atg13, and p62 levels, while downregulated Atg5 levels (Fig. 3c, Fig. 4a), but had no significant effects on cardiac function, hypertrophy, and myocyte apoptosis compared to WT controls (Figs. 1, 2, 3), These finding suggest that ATGL KO-mediated 
4Fig. 6 Blocking proteasome activity reverses PTEN stability and activation of autophagy in ATGL KO mice and cardiomyocytes. a, WT and ATGL KO mice were subjected to sham or TAC operation for 4 weeks. Measurement of the proteasome caspase-like, trypsin-like, and chymotrypsin-like activities in the heart $(n=8)$. b, Immunoblotting of the proteasome catalytic subunits ( $\beta 1 \mathrm{i}, \beta 2 \mathrm{i}$, and $\beta 5 \mathrm{i}$ ) in the heart (left), and quantification of the relative protein levels (right, $n=4$ ). c, Immunoblotting of PTEN, p-AKT, AKT, Sirt1 and GAPDH in the heart (left). Quantification of the relative protein levels (right, $n=4)$. d, WT and ATGL KO mice were subjected to TAC operation and co-treated with VO-OHpic (10 mg/kg daily) or epoxomicin $(2.9 \mathrm{mg} / \mathrm{kg}$ daily) continuously for 4 weeks. Immunoblotting analysis of PTEN, p-mTOR, mTOR, LC3, and GAPDH in the heart (left). Quantification of the relative protein levels (right, $n=4$ ). e, Neonatal rat cardiomyocytes (NRCMs) were co-infected with adenovirus containing mRFPGFP-LC3 and Ad-siRNA-control or Ad-siRNA-ATGL, and then treated with Ang II $(100 \mathrm{nM})$ in the presence or absence of VO-OHpic $(50 \mathrm{nM})$ or epoxomicin $(100 \mathrm{nM})$ continuously for $48 \mathrm{~h}$. Immunofluorescence images (left) and the numbers of autophagosomes (yellow) and autolysosomes (red) in each condition (right, 15-20 cells per group) were quantified $(n=5$ independent experiments). Scale bar: $20 \mu \mathrm{m}$. Data are presented as mean \pm SEM, and $n$ represents number of animals

activation of apoptosis- and autophagy-related signals lead to cardiac dysfunction during pressure overload, but not under basal condition.

Previous studies indicated that ATGL stimulates autophagy/lipophagy by interacting with LC3 or by enhancing Sirt1 activity in the liver or adipose tissues (Martinez-Lopez et al. 2016; Salatzki et al. 2018). Sirt1 activates autophagy through deacetylation and activation of multiple key autophagy genes (Atg5, Atg7, and Atg8) (Lee et al. 2008). However, our results indicated that ATGL KO did not significantly affect Sirt1 protein level in the heart tissues after sham or TAC surgery (Fig. 6c), suggesting that Sirt1 does not participate in inhibition of autophagy in ATGL KO hearts. Interestingly, PTEN/AKT/mTOR signaling critically regulates autophagy via ULK1 phosphorylation (Kim et al. 2018; Shi et al. 2020). PTEN is a key negative regulator of AKT-mTOR and AMPK signaling pathways, which exerts a critical role in the regulation of myocardial hypertrophic remodeling. Its stability is mainly regulated by the proteasome catalytic subunits especially $\beta 1 \mathrm{i}, \beta 2 \mathrm{i}$ and $\beta 5 \mathrm{i}$ (Chen et al. 2019, Li et al. 2018; Xu et al. 2014; Zou et al. 2019). In this study, ATGL KO markedly enhanced the expressions and activities of the proteasome catalytic subunits and PTEN degradation (Fig. 6a-c). Conversely, treatment of ATGL KO mice with epoxomicin significantly upregulated PTEN protein level while suppressing AKT/mTOR signaling, leading to autophagic activation and attenuation of cardiac hypertrophic remodeling after TAC operation. However, these actions were markedly reversed by the PTEN inhibitor, VO-OHpic (Fig. 6d-e, Fig. 7). These findings indicate that ATGL KO inhibits autophagy likely through the proteasome-PTEN-mTOR signaling pathway.

Cardiac oxidative stress, inflammation, and apoptosis are involved in hypertrophy as well as HF pathogeneses. In cardiomyocytes, ATGL has been shown to suppress cardiac NADPH oxidase activities, accompanied by downregulation of NADPH oxidase isoforms and pro-inflammatory mediators. These effects are probably due to reduced cardiac PPAR $\alpha$-mediated regulation of antioxidant enzymes and anti-inflammatory effects in ATGL KO mice (Haemmerle et al. 2011; Schrammel et al. 2013; Vegliante et al. 2018). Altered lipid droplets containing cell-type specific surfacebinding proteins such as perilipin 5 have been shown to induce oxidative and inflammatory responses (Kuramoto et al. 2012). Interestingly, gp91ds-tat (a selective NADPH oxidase inhibitor) and MnTBAP (antioxidant SOD mimetic) ameliorated oxidative stress in WT and ATGL KO heart homogenates. However, due to insufficient reactivity, quality, or composition, systemic treatment with MnTBAP aggravates cardiac oxidative stress in ATGL KO mice (Batinic-Haberle et al. 2009; Reboucas et al. 2008; Schrammel et al. 2013). Studies should investigate the protective effects of NADPH oxidase inhibitors such as gp91ds-tat or antioxidants such as apocynin in oxidative stress in ATGL KO mice (Liu et al. 2010). Global ATGL KO or ATGL inhibition with atglistatin attenuates AMPK phosphorylation in adipose tissues (MacPherson et al. 2016). Consistently, we found that ATGL KO suppressed AMPK activation in the heart following TAC or sham operation (Fig. 3d). However, there are several limitations in the presents study: The precise mechanisms through which ATGL regulates the activation of AMPK, NF- $\kappa \mathrm{B}$, and NOX signals, the expression and activity of the proteasome catalytic subunits in the heart should be evaluated further. Moreover, ATGL regulates autophagy in cardiomyocyte-specific ATGL knockout mice should be investigated.

In conclusion, our results reveal a novel mechanism for ATGL to prevent pressure overload-induced cardiac hypertrophy and HF. ATGL KO increases the 
a

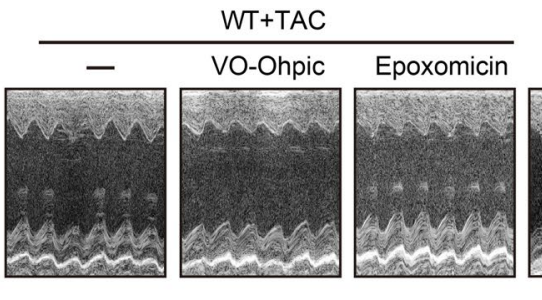

b

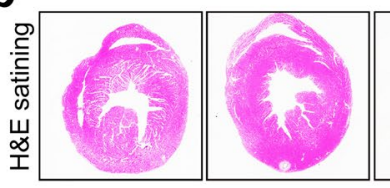

C

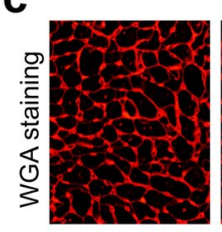

d
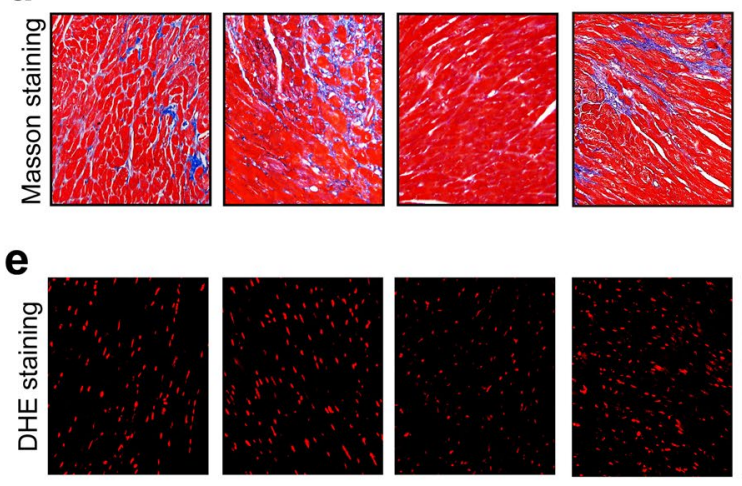

f

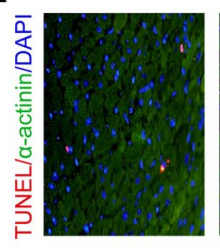

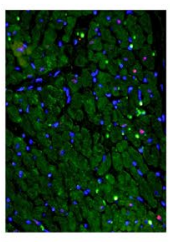
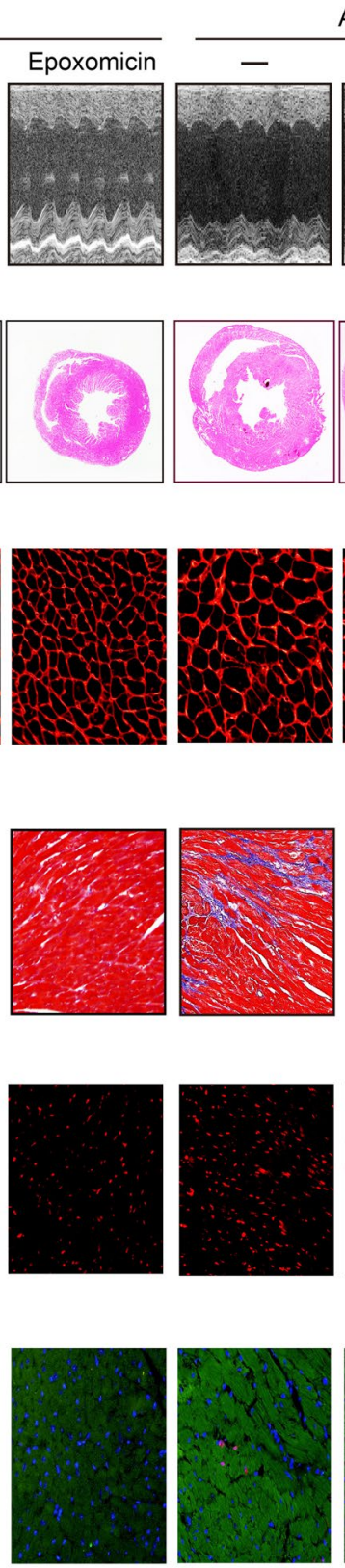

ATGL KO+TAC
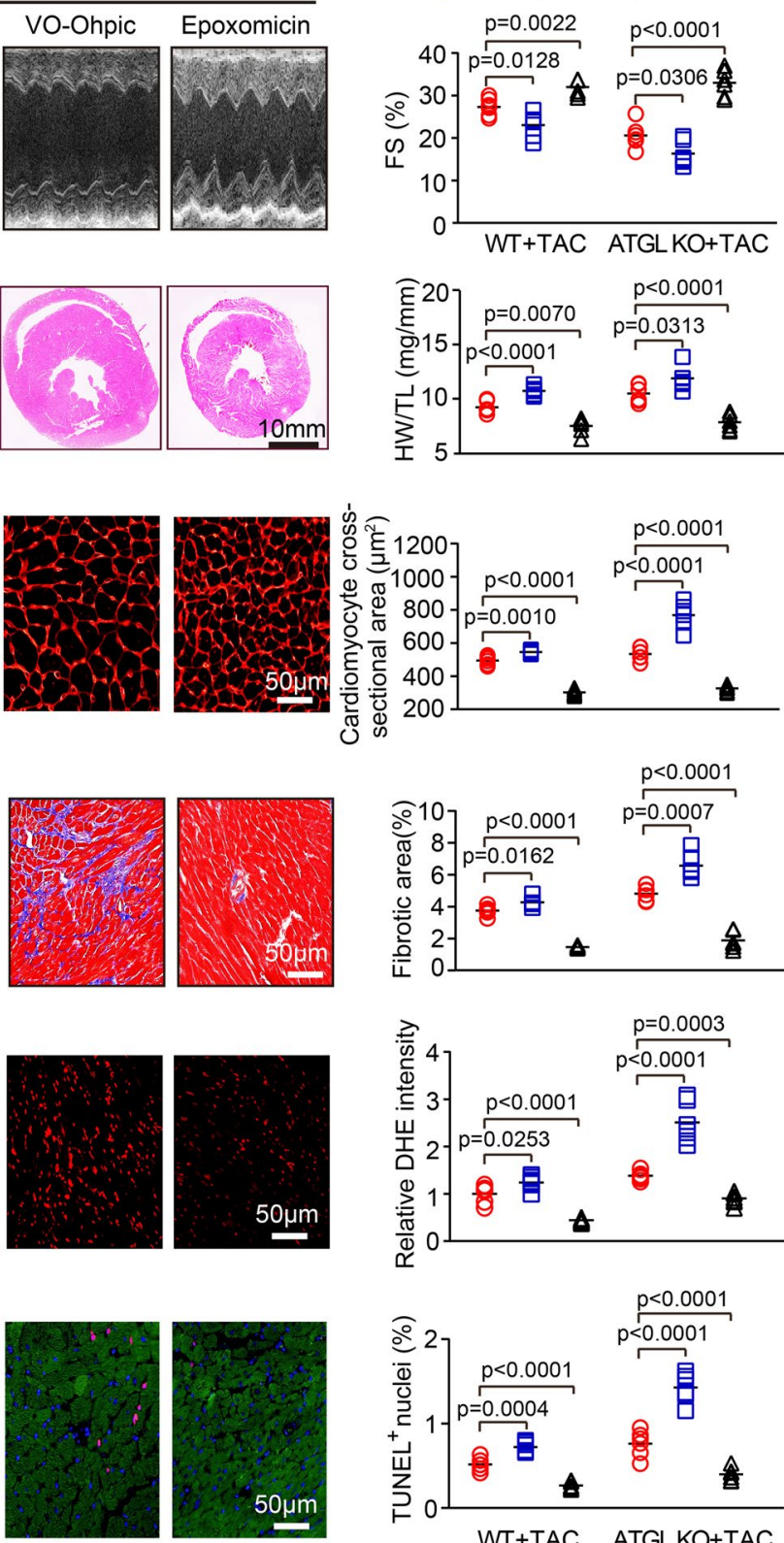
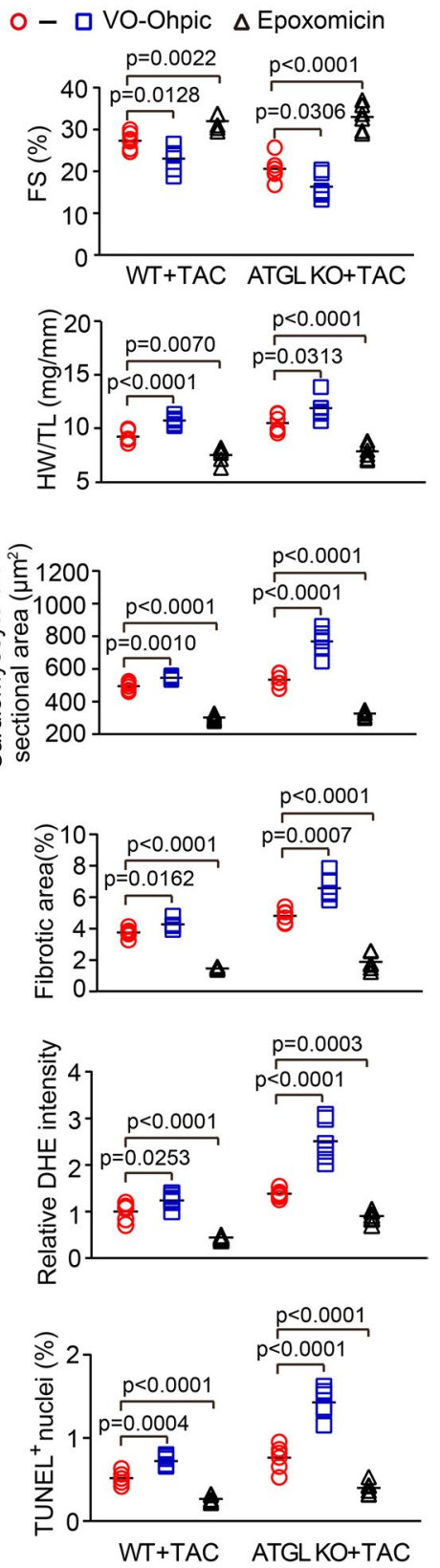

Fig. 7 Blocking proteasome activity reverses TAC-induced cardiac dysfunction and hypertrophic remodeling. a, WT and ATGL KO mice were subjected to TAC operation and co-treated with VO-OHpic (10 mg/kg daily) or epoxomicin $(2.9 \mathrm{mg} / \mathrm{kg}$ daily) continuously for 4 weeks. Echocardiographic measurement of LV chamber (left), and the calculation of LV FS\% (right, $n=6$ ). b, Representative H\&E staining of heart sections (left). Scale bar: $10 \mathrm{~mm}$. The ratio of HW/TL (right, $n=6$ ). c, TRITC-labeled WGA staining of heat sections (left).

proteasome level and activity, which promotes PTEN degradation and activation of AKT-mTOR signaling,

Quantification of cross-sectional area of myocytes (right, $n=6$, 150-200 cells counted per sample). Scale bar: $50 \mu \mathrm{m}$. d, Masson's trichrome staining of heat sections (left). Quantification of fibrotic area (right, $n=6$ ). Scale bar: $50 \mu \mathrm{m}$. e, DHE staining of heart sections (left). Quantification of the relative superoxide production (right, $n=6$ ). Scale bar: $50 \mu \mathrm{m}$. f, TUNEL staining (left). Quantification of the TUNEL-positive nuclei (right, $n=6$ ). Scale bar: $50 \mu \mathrm{m}$. Data are presented as mean \pm SEM, and $n$ represents number of animals

resulting in suppression of autophagy and cardiac hypertrophy and HF. 
Abbreviations ATGL: Adipose triglyceride lipase; TAC: Transverse aortic constriction; TAG: Triacylglycerol; EF: Left ventricle ejection fraction; FS: Left ventricle fractional shortening; LVSP: Left ventricle systolic pressure; SV: Stroke volume; Ea: Arterial elastance (measure of ventricular afterload); $-\mathrm{dp} / \mathrm{dt}$ : Maximal rate of pressure decline (diastolic indexes).; Tau: Relaxation time constant

Author contribution X.H., Y.L.Z., and Q.Y.L. conceived of the experiments, the acquisition of the data and analysis, and interpreted the data. X.H. and Y.L.Z. participated in the statistical analysis of the primary data. H.H.L. and S.B.G drafted the manuscript. H.H.L. provided funding to support the study and supervised the study. All authors approved the final version of the manuscript.

Funding This work was supported by grants from China National Natural Science Funds (82030009).

Data availability The datasets generated during and/or analyzed during the current study are available from the corresponding author on reasonable request.

Code availability Not applicable.

\section{Declarations}

Ethics approval and consent to participate The investigation was approved by the Animal Care and Use Committee of Beijing Chao-Yang Hospital of Capital Medical University and conformed to the Guide for the Care and Use of Laboratory Animals published by the U.S. National Institutes of Health (NIH Publication No.85-23, revised 1996).

Consent for publication All authors have agreed to publish this manuscript.

Conflict of interest The authors declare no competing interests.

Open Access This article is licensed under a Creative Commons Attribution 4.0 International License, which permits use, sharing, adaptation, distribution and reproduction in any medium or format, as long as you give appropriate credit to the original author(s) and the source, provide a link to the Creative Commons licence, and indicate if changes were made. The images or other third party material in this article are included in the article's Creative Commons licence, unless indicated otherwise in a credit line to the material. If material is not included in the article's Creative Commons licence and your intended use is not permitted by statutory regulation or exceeds the permitted use, you will need to obtain permission directly from the copyright holder. To view a copy of this licence, visit http://creativecommons.org/licenses/by/4.0/.

\section{References}

Batinic-Haberle I, Cuzzocrea S, Reboucas JS, Ferrer-Sueta G, Mazzon E, Di Paola R, et al. Pure MnTBAP selectively scavenges peroxynitrite over superoxide: comparison of pure and commercial MnTBAP samples to MnTE-2-PyP in two models of oxidative stress injury, an SOD-specific Escherichia coli model and carrageenan-induced pleurisy. Free Radic Biol Med. 2009;46(2):192-201.

Bu W, Hao X, Yang T, Wang J, Liu Q, Zhang X, et al. Autophagy contributes to the maintenance of genomic integrity by reducing oxidative stress. Oxid Med Cell Longev. 2020;2020:2015920.

Cao HJ, Fang J, Zhang YL, Zou LX, Han X, Yang J, et al. Genetic ablation and pharmacological inhibition of immunosubunit beta5i attenuates cardiac remodeling in deoxycorticosterone-acetate (DOCA)-salt hypertensive mice. J Mol Cell Cardiol. 2019;137:34-45.

Chen C, Zou LX, Lin QY, Yan X, Bi HL, Xie X, et al. Resveratrol as a new inhibitor of immunoproteasome prevents PTEN degradation and attenuates cardiac hypertrophy after pressure overload. Redox Biol. 2019;203:90-401.

Deng H, Ma LL, Kong FJ, Qiao Z. Distinct phenotypes induced by different degrees of transverse aortic constriction in C57BL/6N mice. Front Cardiovasc Med. 2021;8:641272.

Foryst-Ludwig A, Kreissl MC, Benz V, Brix S, Smeir E, Ban Z, et al. Adipose Tissue Lipolysis Promotes Exercise-induced Cardiac Hypertrophy Involving the Lipokine C16:1n7Palmitoleate. J Biol Chem. 2015;290(39):23603-15.

Ganley IG, Lam du H, Wang J, Ding X, Chen S, Jiang X. ULK1.ATG13.FIP200 complex mediates mTOR signaling and is essential for autophagy. J Biol Chem. 2009;284(18):12297-305.

Gao H, Feng XJ, Li ZM, Li M, Gao S, He YH, et al. Downregulation of adipose triglyceride lipase promotes cardiomyocyte hypertrophy by triggering the accumulation of ceramides. Arch Biochem Biophys. 2015;56:576-88.

Haemmerle G, Lass A, Zimmermann R, Gorkiewicz G, Meyer C, Rozman J, et al. Defective lipolysis and altered energy metabolism in mice lacking adipose triglyceride lipase. Science. 2006;312(5774):734-7.

Haemmerle G, Moustafa T, Woelkart G, Buttner S, Schmidt A, van de Weijer T, et al. ATGL-mediated fat catabolism regulates cardiac mitochondrial function via PPAR-alpha and PGC-1. Nat Med. 2011;17(9):1076-85.

Han D, Zhang QY, Zhang YL, Han X, Guo SB, Teng F, et al. Gallic acid ameliorates angiotensin II-induced atrial fibrillation by inhibiting immunoproteasome- mediated PTEN degradation in mice. Front Cell Dev Biol. 2020;8:594683.

Heineke J, Molkentin JD. Regulation of cardiac hypertrophy by intracellular signalling pathways. Nat Rev Mol Cell Biol. 2006;7(8):589-600.

Hirano K, Ikeda Y, Zaima N, Sakata Y, Matsumiya G. Triglyceride deposit cardiomyovasculopathy. N Engl J Med. 2008;359(22):2396-8.

Kienesberger PC, Pulinilkunnil T, Sung MM, Nagendran J, Haemmerle G, Kershaw EE, et al. Myocardial ATGL overexpression decreases the reliance on fatty acid oxidation and protects against pressure overload-induced cardiac dysfunction. Mol Cell Biol. 2012;32(4):740-50. 
Kim JH, Choi TG, Park S, Yun HR, Nguyen NNY, Jo YH, et al. Mitochondrial ROS-derived PTEN oxidation activates PI3K pathway for mTOR-induced myogenic autophagy. Cell Death Differ. 2018;25(11):1921-37.

Kintscher U, Foryst-Ludwig A, Haemmerle G, Zechner R. The Role of Adipose Triglyceride Lipase and Cytosolic Lipolysis in Cardiac Function and Heart Failure. Cell Rep Med. 2020;1(1):100001.

Kuramoto K, Okamura T, Yamaguchi T, Nakamura TY, Wakabayashi $\mathrm{S}$, Morinaga $\mathrm{H}$, et al. Perilipin 5, a lipid dropletbinding protein, protects heart from oxidative burden by sequestering fatty acid from excessive oxidation. J Biol Chem. 2012;287(28):23852-63.

Lee IH, Cao L, Mostoslavsky R, Lombard DB, Liu J, Bruns NE, et al. A role for the NAD-dependent deacetylase Sirt1 in the regulation of autophagy. Proc Natl Acad Sci USA. 2008;105(9):3374-9.

Li J, Wang S, Bai J, Yang XL, Zhang YL, Che YL, et al. Novel Role for the Immunoproteasome Subunit PSMB10 in Angiotensin II-Induced Atrial Fibrillation in Mice. Hypertension. 2018;71(5):866-76.

Li L, Xu J, He L, Peng L, Zhong Q, Chen L, et al. The role of autophagy in cardiac hypertrophy. Acta Biochim Biophys Sin (shanghai). 2016;48(6):491-500.

Liao J, An X, Yang X, Lin QY, Liu S, Xie Y, et al. Deficiency of LMP10 attenuates diet-induced atherosclerosis by inhibiting macrophage polarization and inflammation in apolipoprotein E deficient mice. Front Cell Dev Biol. 2020;8:592048.

Lin QY, Zhang YL, Bai J, Liu JQ, Li HH. VEGF-C/VEGFR-3 axis protects against pressure-overload induced cardiac dysfunction through regulation of lymphangiogenesis. Clin Transl Med. 2021;11(3):e374.

Liu J, Zhou J, An W, Lin Y, Yang Y, Zang W. Apocynin attenuates pressure overload-induced cardiac hypertrophy in rats by reducing levels of reactive oxygen species. Can J Physiol Pharmacol. 2010;88(7):745-52.

MacPherson RE, Dragos SM, Ramos S, Sutton C, FrendoCumbo S, Castellani L, et al. Reduced ATGL-mediated lipolysis attenuates beta-adrenergic-induced AMPK signaling, but not the induction of PKA-targeted genes, in adipocytes and adipose tissue. Am J Physiol Cell Physiol. 2016;311(2):C269-76.

Martinez-Lopez N, Garcia-Macia M, Sahu S, Athonvarangkul D, Liebling E, Merlo P, et al. Autophagy in the CNS and Periphery Coordinate Lipophagy and Lipolysis in the Brown Adipose Tissue and Liver. Cell Metab. 2016;23(1):113-27.

Meng L, Mohan R, Kwok BH, Elofsson M, Sin N, Crews CM. Epoxomicin, a potent and selective proteasome inhibitor, exhibits in vivo antiinflammatory activity. Proc Natl Acad Sci USA. 1999;96(18):10403-8.

Nakai A, Yamaguchi O, Takeda T, Higuchi Y, Hikoso S, Taniike M, et al. The role of autophagy in cardiomyocytes in the basal state and in response to hemodynamic stress. Nat Med. 2007;13(5):619-24.

Reboucas JS, Spasojevic I, Batinic-Haberle I. Quality of potent $\mathrm{Mn}$ porphyrin-based SOD mimics and peroxynitrite scavengers for pre-clinical mechanistic/therapeutic purposes. J Pharm Biomed Anal. 2008;48(3):1046-9.

Roe ND, Xu X, Kandadi MR, Hu N, Pang J, Weiser-Evans $\mathrm{MC}$, et al. Targeted deletion of PTEN in cardiomyocytes renders cardiac contractile dysfunction through interruption of Pink1-AMPK signaling and autophagy. Biochim Biophys Acta. 2015;1852(2):290-8.

Sai E, Shimada K, Yokoyama T, Hiki M, Sato S, Hamasaki $\mathrm{N}$, et al. Myocardial triglyceride content in patients with left ventricular hypertrophy: comparison between hypertensive heart disease and hypertrophic cardiomyopathy. Heart Vessels. 2017;32(2):166-74.

Salatzki J, Foryst-Ludwig A, Bentele K, Blumrich A, Smeir E, Ban Z, et al. Adipose tissue ATGL modifies the cardiac lipidome in pressure-overload-induced left ventricular failure. PLoS Genet. 2018;14(1):e1007171.

Sathyanarayan A, Mashek MT, Mashek DG. ATGL Promotes Autophagy/Lipophagy via SIRT1 to Control Hepatic Lipid Droplet Catabolism. Cell Rep. 2017;19(1):1-9.

Schrammel A, Mussbacher M, Winkler S, Haemmerle G, Stessel H, Wolkart G, et al. Cardiac oxidative stress in a mouse model of neutral lipid storage disease. Biochim Biophys Acta. 2013;1831(11):1600-8.

Shi YX, He YJ, Zhou Y, Li HK, Yang D, Li RY, et al. LSD1 negatively regulates autophagy in myoblast cells by driving PTEN degradation. Biochem Biophys Res Commun. 2020;522(4):924-30.

Takahara S, Ferdaoussi M, Srnic N, Maayah ZH, Soni S, Migglautsch AK, et al. Inhibition of ATGL in adipose tissue ameliorates isoproterenol-induced cardiac remodeling by reducing adipose tissue inflammation. Am $\mathbf{J}$ Physiol Heart Circ Physiol. 2021;320(1):H432-46.

Thiele A, Luettges K, Ritter D, Beyhoff N, Smeir E, Grune J, et al. Pharmacological inhibition of adipose tissue Adipose Triglyceride Lipase (ATGL) by Atglistatin prevents catecholamine-induced myocardial damage. Cardiovasc Res. 2021. https://doi.org/10.1093/cvr/cvab182.

Trites MJ, Clugston RD. The role of adipose triglyceride lipase in lipid and glucose homeostasis: lessons from transgenic mice. Lipids Health Dis. 2019;18(1):204.

Vegliante R, Di Leo L, Ciccarone F, Ciriolo MR. Hints on ATGL implications in cancer: beyond bioenergetic clues. Cell Death Dis. 2018;9(3):316.

Wang X, Cui T. Autophagy modulation: a potential therapeutic approach in cardiac hypertrophy. Am J Physiol Heart Circ Physiol. 2017;313(2):H304-19.

Xie X, Bi HL, Lai S, Zhang YL, Li N, Cao HJ, et al. The immunoproteasome catalytic beta5i subunit regulates cardiac hypertrophy by targeting the autophagy protein ATG5 for degradation. Sci Adv. 2019;5(5):eaau0495.

Xie X, Wang HX, Li N, Deng YW, Bi HL, Zhang YL, et al. Selective inhibition of the immunoproteasome beta5i prevents PTEN degradation and attenuates cardiac hypertrophy. Front Pharmacol. 2020;11:885.

$\mathrm{Xu}$ X, Roe ND, Weiser-Evans MC, Ren J. Inhibition of mammalian target of rapamycin with rapamycin reverses hypertrophic cardiomyopathy in mice with 
cardiomyocyte-specific knockout of PTEN. Hypertension. 2014;63(4):729-39.

Yan W, Dong ZC, Wang JJ, Zhang YL, Wang HX, Zhang B, et al. Deficiency of the immunoproteasome LMP10 subunit attenuates angiotensin II-induced cardiac hypertrophic remodeling via autophagic degradation of gp130 and IGF1R. Front Physiol. 2020;11:625.

Yan X, Zhang YL, Zhang L, Zou LX, Chen C, Liu Y, et al. Gallic Acid Suppresses Cardiac Hypertrophic Remodeling and Heart Failure. Mol Nutr Food Res. 2019;63(5):e1800807.

Yang HJ, Kong B, Shuai W, Zhang JJ, Huang H. MD1 deletion exaggerates cardiomyocyte autophagy induced by heart failure with preserved ejection fraction through ROS/MAPK signalling pathway. J Cell Mol Med. 2020;24(16):9300-12.

Yang N, Liu X, Niu X, Wang X, Jiang R, Yuan N, et al. Activation of autophagy ameliorates age-related neurogenesis decline and neurodysfunction in adult mice. Stem Cell Rev Rep. 2021. https://doi.org/10.1007/s12015-021-10265-0.

Zhu H, Tannous P, Johnstone JL, Kong Y, Shelton JM, Richardson JA, et al. Cardiac autophagy is a maladaptive response to hemodynamic stress. J Clin Invest. 2007;117(7):1782-93.

Zou LX, Chen C, Yan X, Lin QY, Fang J, Li PB, et al. Resveratrol Attenuates Pressure Overload-Induced Cardiac Fibrosis and Diastolic Dysfunction via PTEN/AKT/Smad2/3 and NF-kappaB Signaling Pathways. Mol Nutr Food Res. 2019;63(24):e1900418.

Publisher's note Springer Nature remains neutral with regard to jurisdictional claims in published maps and institutional affiliations. 